6th International Workshop

on Ring Imaging Cherenkov Counters

15-20 October 2007

Trieste - Italy

\title{
Status and perspectives of vacuum-based photon detectors for single photon detection
}

\author{
Thierry Gys \\ CERN - Geneva - Switzerland
}


- Much more is taking place than what can be covered in 40 minutes!

- The selection criteria were a combination of:

- the speaker's current activities and interests,

- those developments coming from relatively new and near-future R\&D and experiment projects,

- topics which are generally covered in other oral or poster presentations during this Conference,

- topics the illustrations of which were easily accessible, directly via authors, publications and web sites. 


\section{Broad range of requirements}

- Photon detection

- Total surface: $\sim 1 \mathrm{~mm}^{2}$ to $\sim 1 \mathrm{~m}^{2}$

- Granularity: $~ 10 \mu \mathrm{m}$ to $\geq 10 \mathrm{~mm}$

- Active area coverage: $\geq 50 \%$

- Single-photon sensitivity over a broad spectral range (near UV to infrared)

- Environment

- Magnetic field: $1 \mathrm{mT} \leq \mathrm{B} \leq 4 \mathrm{~T}$

(axial and/or transverse)

- Radiation dose: $100 \mathrm{kRad} /$ year (charged particles)

$$
510^{10} \mathrm{~cm}^{-2} \text { (neutrons) }
$$

$\checkmark$ Read-out

- Maximum occupancy: $\leq 10 \%$

- Intrinsic speed: $\leq n s$

- Signal jitter: 10 ps to 10 ns

- Signal rate: $\sim 1 \mathrm{~Hz}$ to $\sim 100 \mathrm{Mhz}$

- Read-out rate: $\sim 1 \mathrm{~Hz}$ to $\sim 1 \mathrm{MHz}$ 
This overview will mainly focus on position-sensitive single-photon detectors:

- Multi-anode Photon Multiplier Tubes

- Micro Channel Plate Photon Multiplier Tubes

- Hybrid Photon Detectors

- Electron-bombarded CCD's or alternatives

- Etc. 


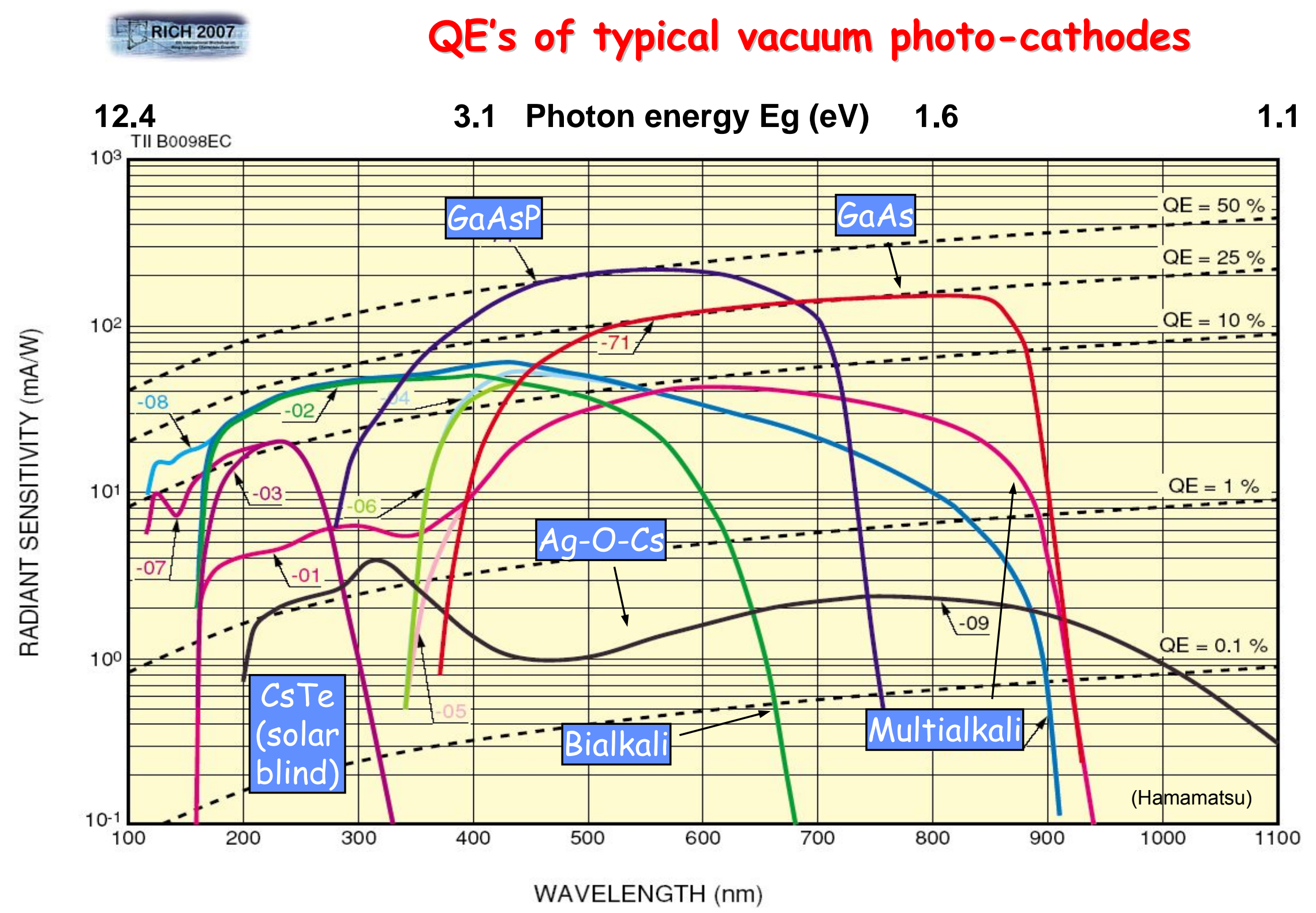

Bialkali: SbKCs, SbRbCs Multialkali: $\mathrm{SbNa}_{2} \mathrm{KCS}$ (alkali metals have low work function) T. Gys - Vacuum photon detectors - RICH 2007 


\section{Main features}

- Construction

- Metal channel dynodes

- Up to $8 \times 8$ channels $\left(2 \times 2 \mathrm{~mm}^{2}\right.$ each):

- Size: $28 \times 28 \mathrm{~mm}^{2}$;

- Active area $18.1 \times 18.1 \mathrm{~mm}^{2}(41 \%)$;

- Bi-alkali PC:

- $Q E \cong$ typ. $\geq 20 \%$ @ $\lambda_{\max }=400 \mathrm{~nm}$ :

- Gain $\cong 3 \times 10^{5}$

- Fluctuation $\cong \sqrt{ } g_{1} / g_{1}$ typ. 0.60;

- Gain uniformity typ. 1 : 2.5:

- Cross-talk typ. $<1-2 \%$
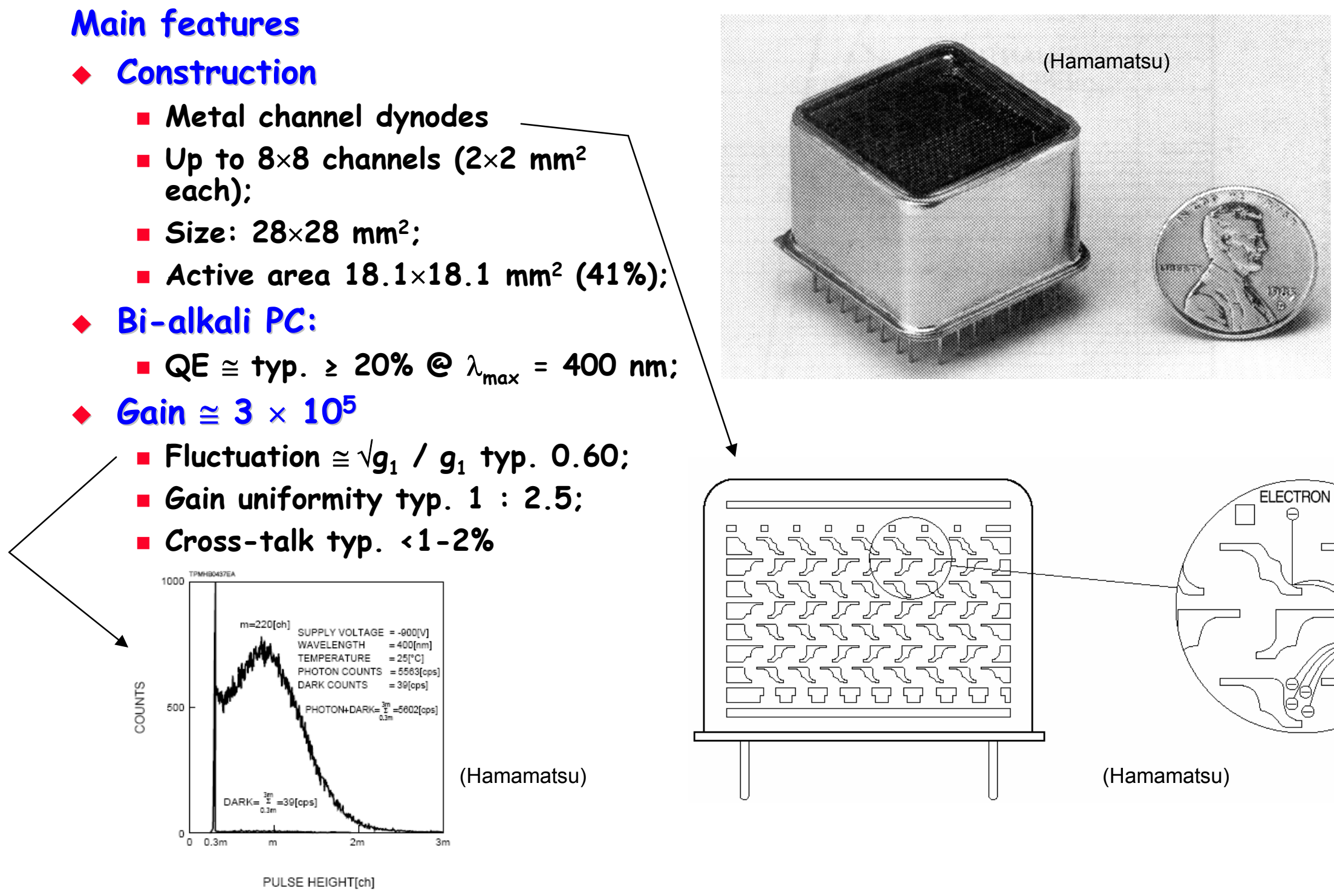

T. Gys - Vacuum photon detectors - RICH 2007 
Hera-B RICH detector

- Requirements

- Rates $1 \mathrm{MHz}$

- Long term stability

- Surface of $\sim 3 \mathrm{~m}^{2}$

- 2 types of MaPMT's:

- 1488 M16 (4.5×4.5 $\mathrm{mm}^{2}$ pads)

- 752 M4 (9×9mm pads)
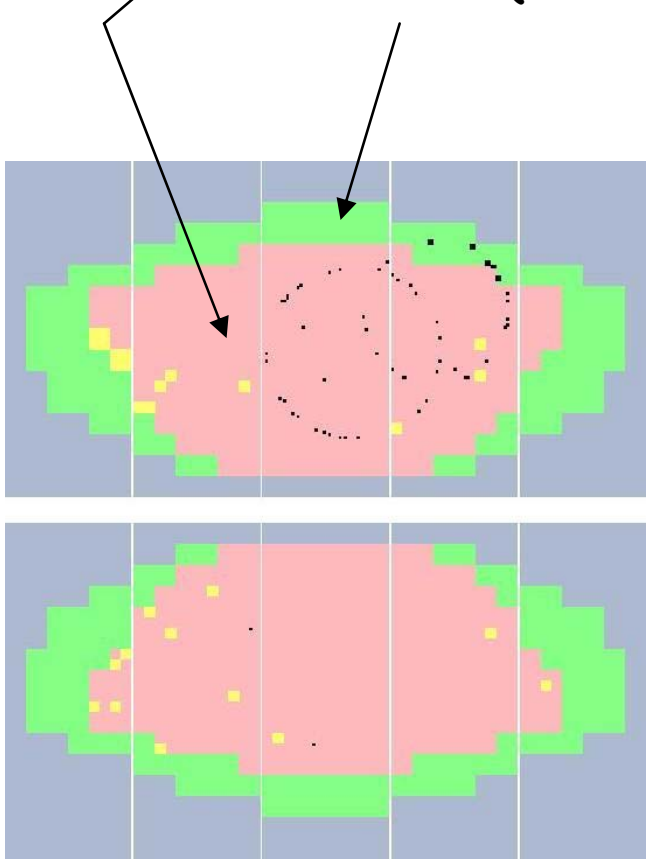

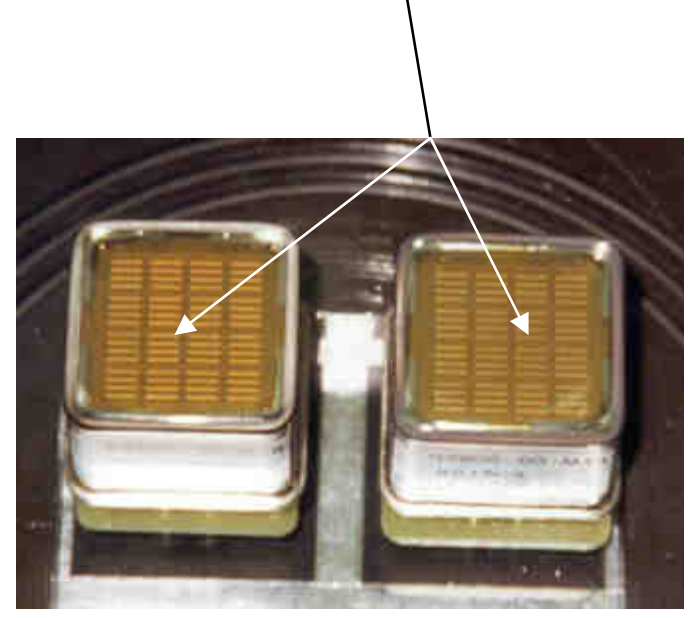

(http://www-hera-b.desy.de/subgroup/detector/rich/)

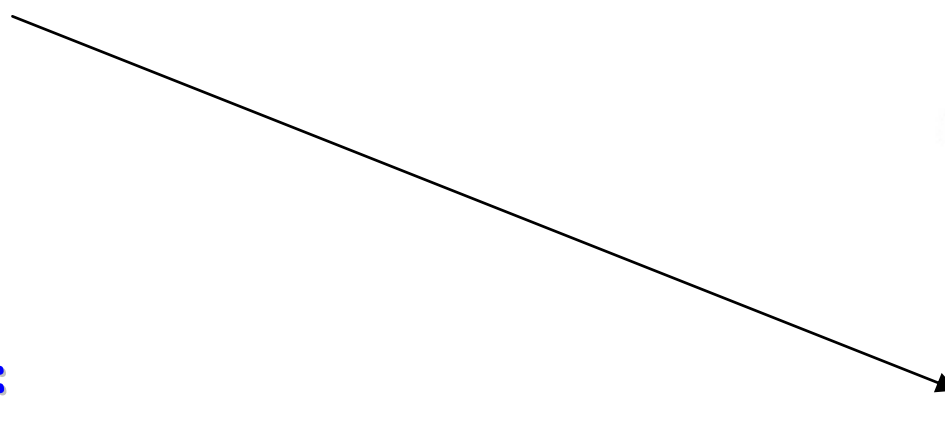

\section{Photon \\ etectors}


Hera-B RICH (cont'd)

- Use lenses to match active area

- $70 \%$ transmission

- drop above $3.5 \mathrm{eV}$ photon energy

- Performance:

- $>98 \%$ single pe efficiency

- $<0.2 \%$ cross-talk

- Quality factor $N_{0}$ :

- average value $42 \mathrm{~cm}^{-1}$

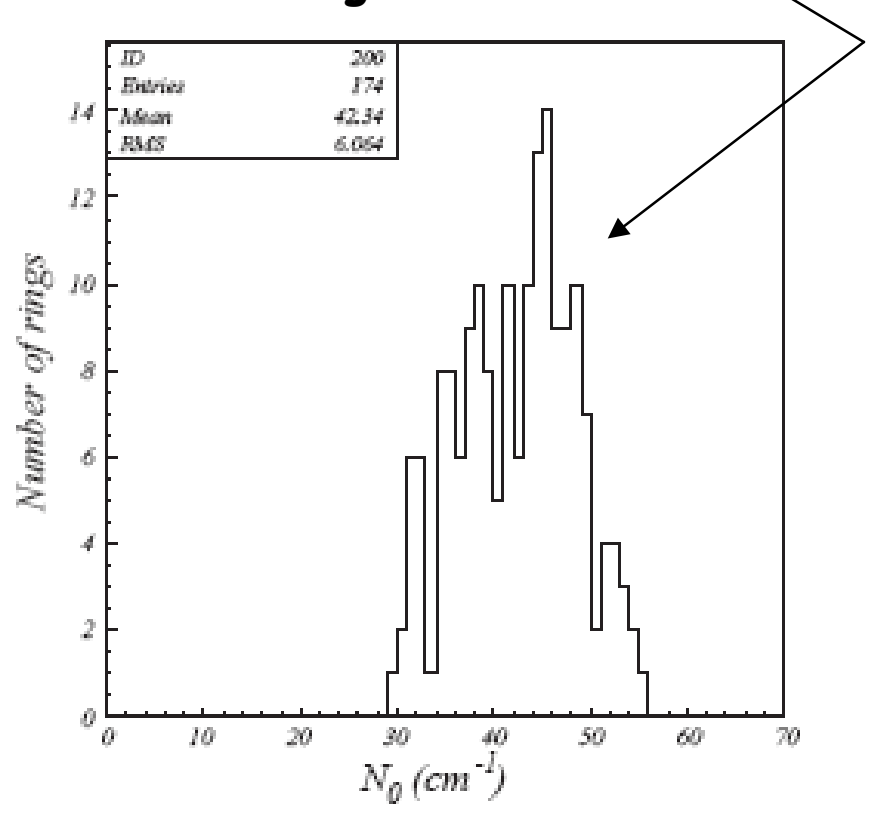

(I. Ariño et al.,

NIM A 516 (2004) 445)

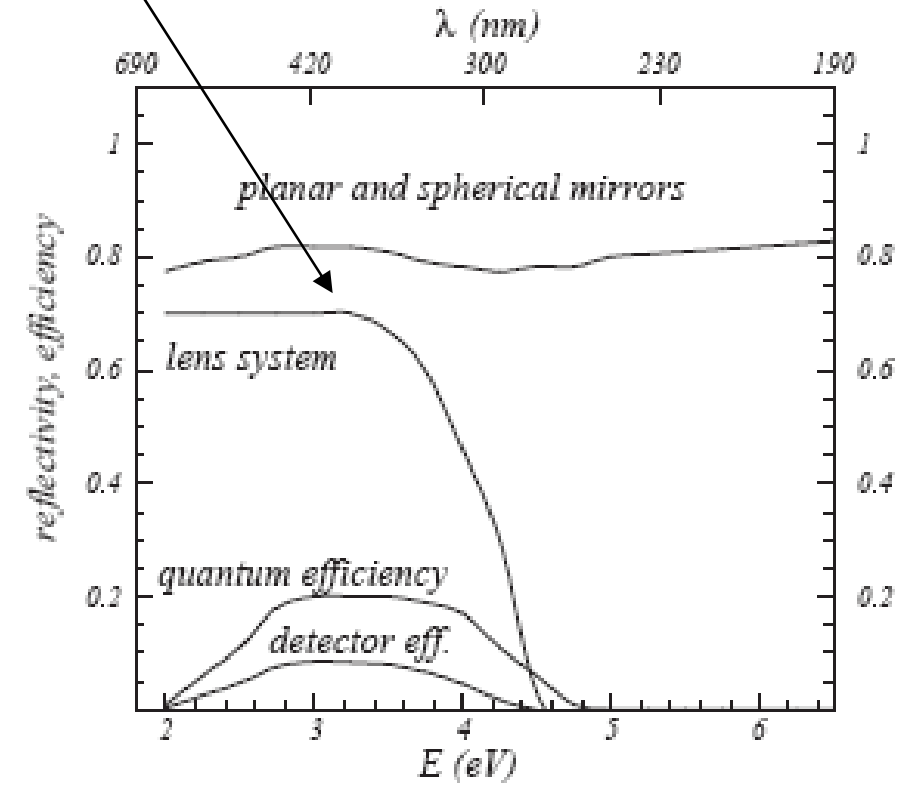

T. Gys - Vacuum photon detectors - RICH 2007 
"Old" Compass RICH1 system

- CSI photo-cathodes - MWPC

- $3 \mu \mathrm{sec}$ memory with Gassiplex chip (now reduced to 400ns with APV25-S1 chip)

- Beam rate $40 \mathrm{MHz}$

- Trigger rate $20 \mathrm{kHz}$
New Compass fast RICH1 (central region)

See contribution of $F$. Tessarotto at this Conference

- 576 M16 MaPMTs (bialkali pc)

- 10nsec time cut

- Beam rate $100 \mathrm{MHz}$

- Trigger rate $100 \mathrm{kHz}$

(http://wwwcompass.cern.ch/compass/)

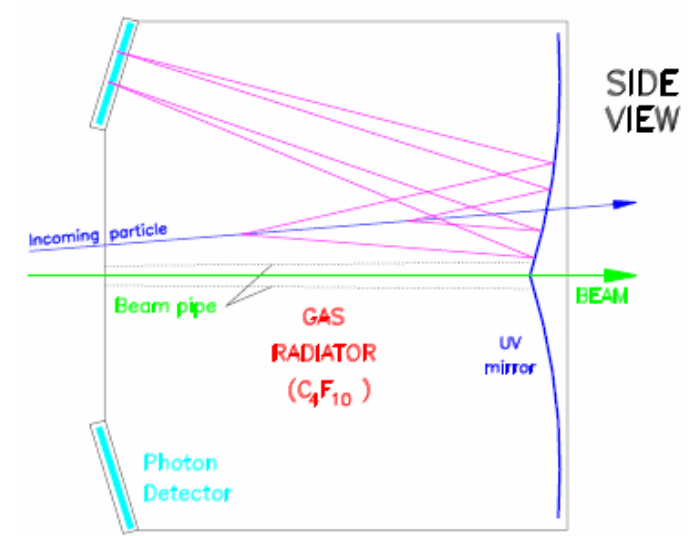

SIDE
VIEW

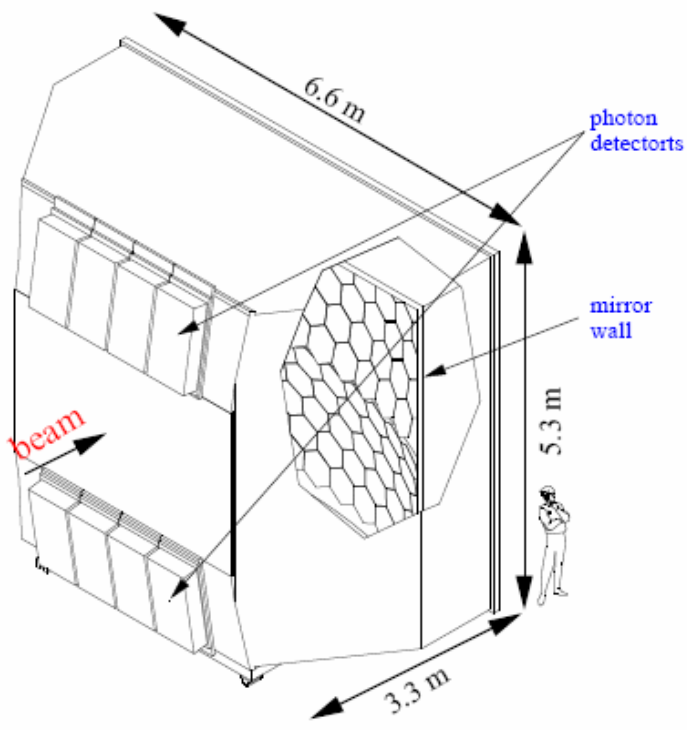

T. Gys - Vacuum photon detectors - RICH 2007 


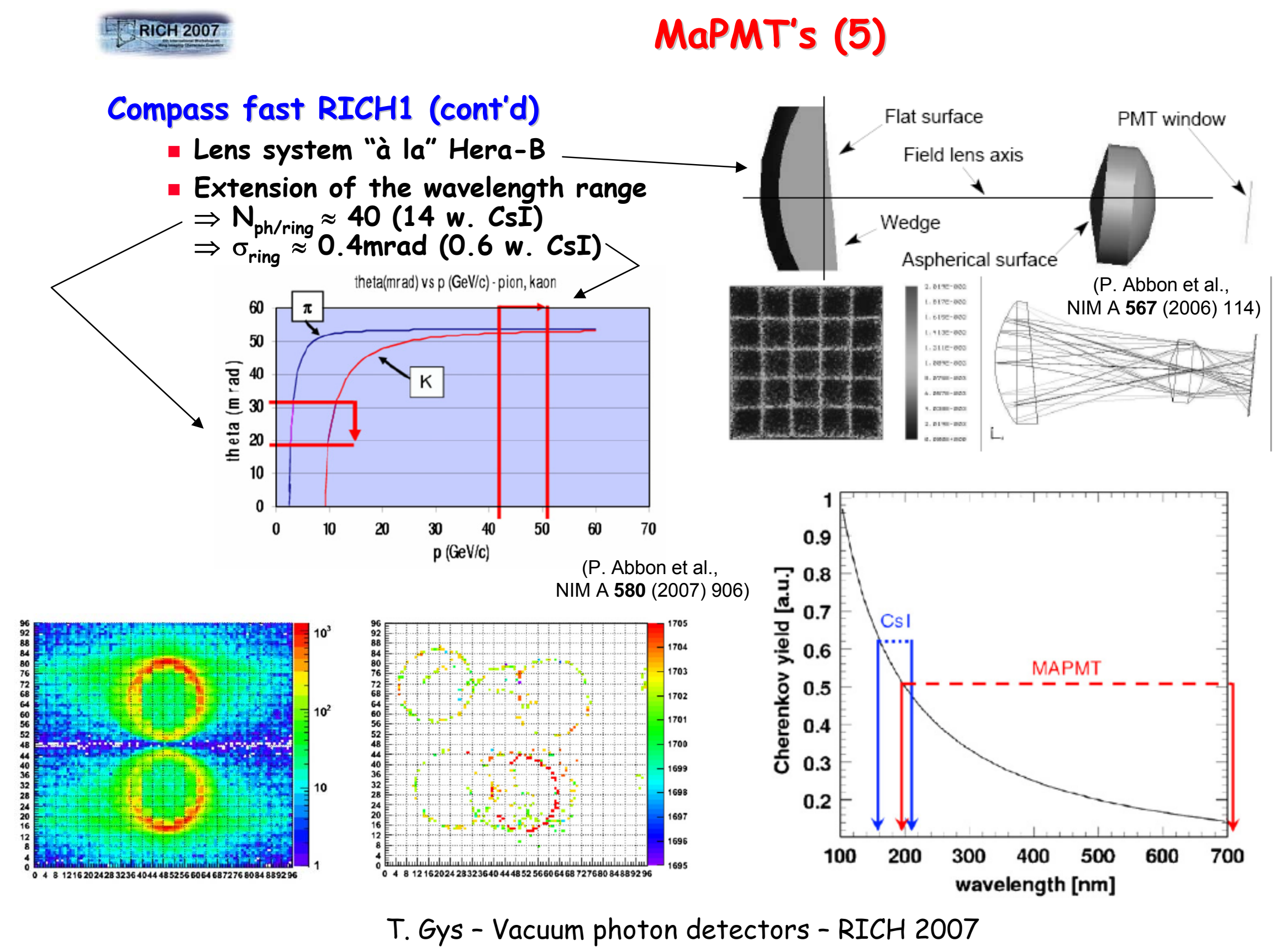




\section{$\mathrm{LHCb}$ pre-shower detector}

- Requirements

- $2 X_{0} \mathrm{~Pb}$ sheets inserted between 2 planes of scintillating tiles - 6000 detector cells

- Hadronic shower rejection threshold 5MIP's, accuracy $0.1 \mathrm{MIP}$, range $100 \mathrm{MIP}$ 's

- MaPMT readout

- typ. 20-30 pe- per MIP

(http://lhcb-calo.web.cern.ch//hcbcalo/html/photos.htm)

- wide linearity range

$\Rightarrow$ low gain $\approx 10^{3}-10^{4}$

- low cross talk « $1 \%$

- ASIC readout chip

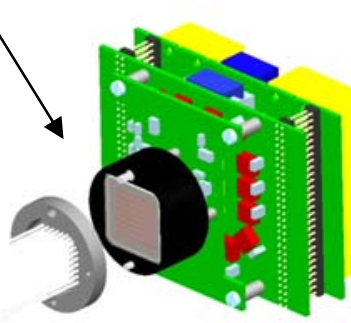

(P. Perret, LHCb-PS EDR)

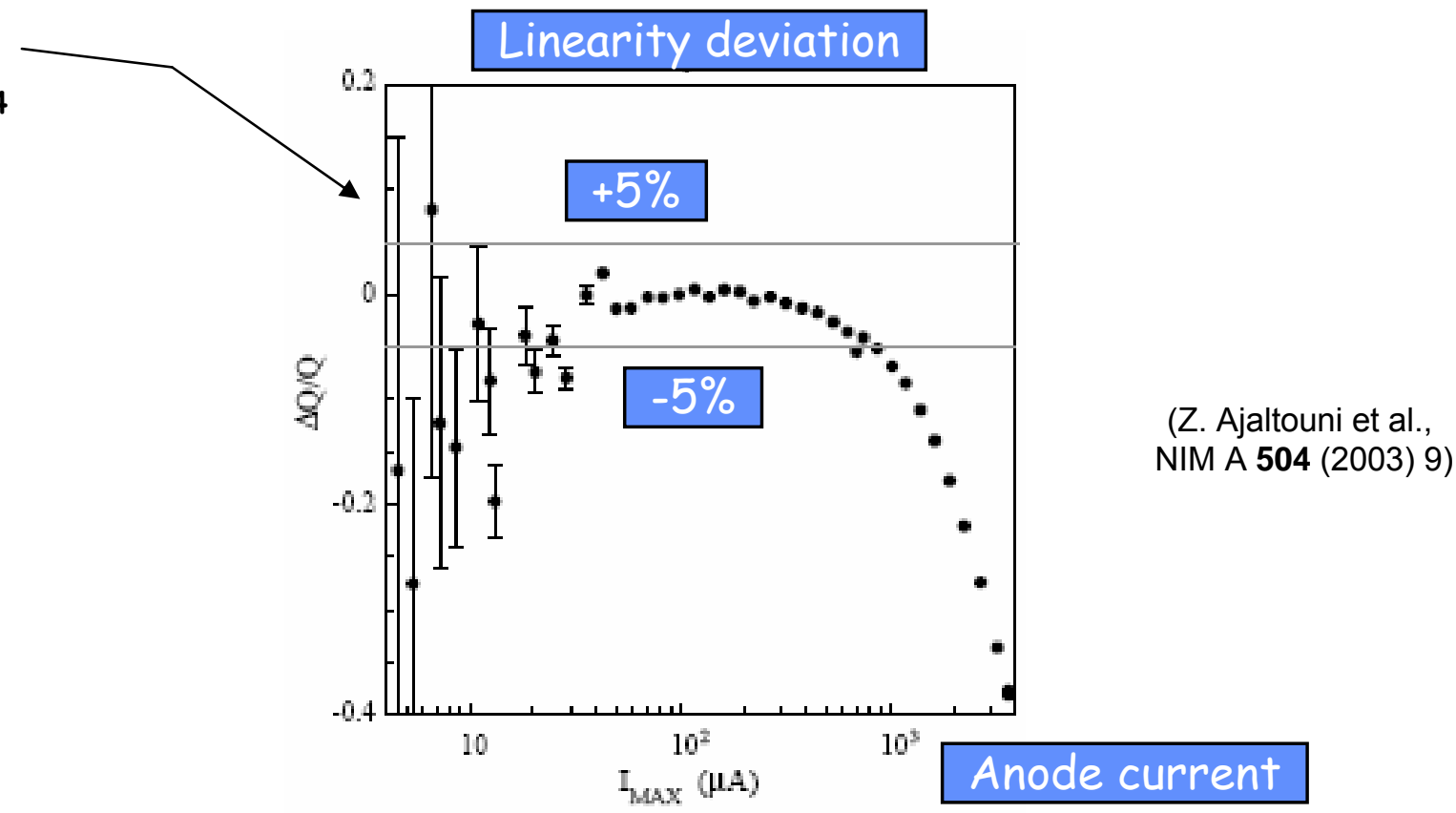

T. Gys - Vacuum photon detectors - RICH 2007 


\section{Flat-panel PMT's}

- Main features

- $8 \times 8$ or $16 \times 16$ channels:

- Compactness:

- Excellent active area ratio (89\%)

- Belle upgrade

See contribution of $T$. Iijima at this Conference

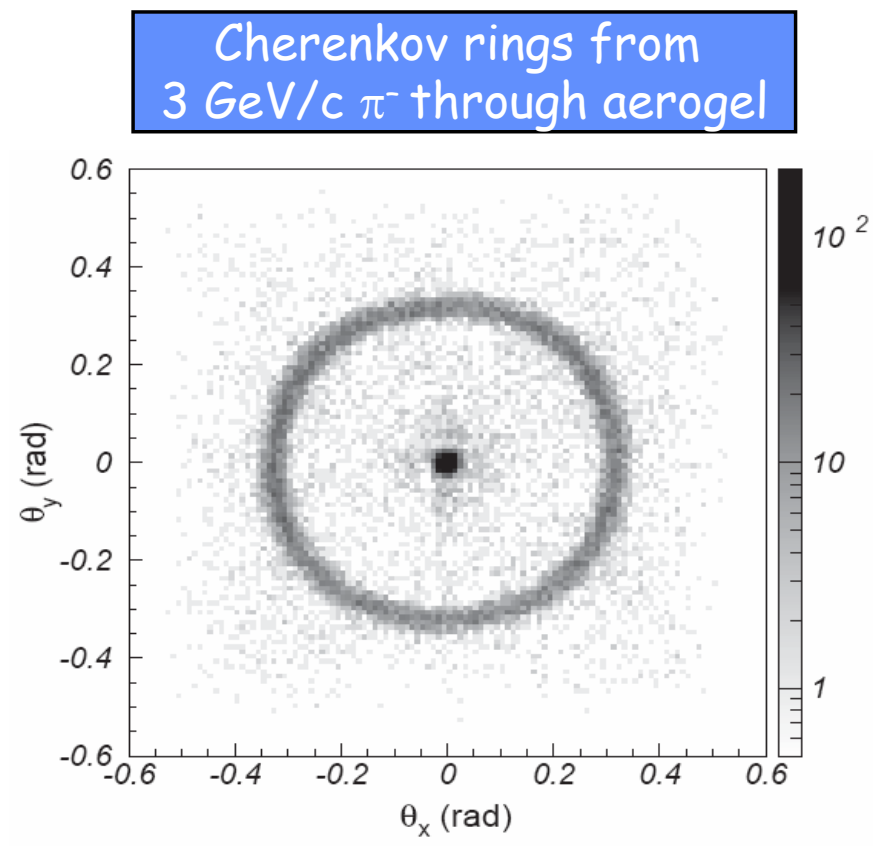

(T. Matsumoto et al., NIM A 521 (2004) 367)
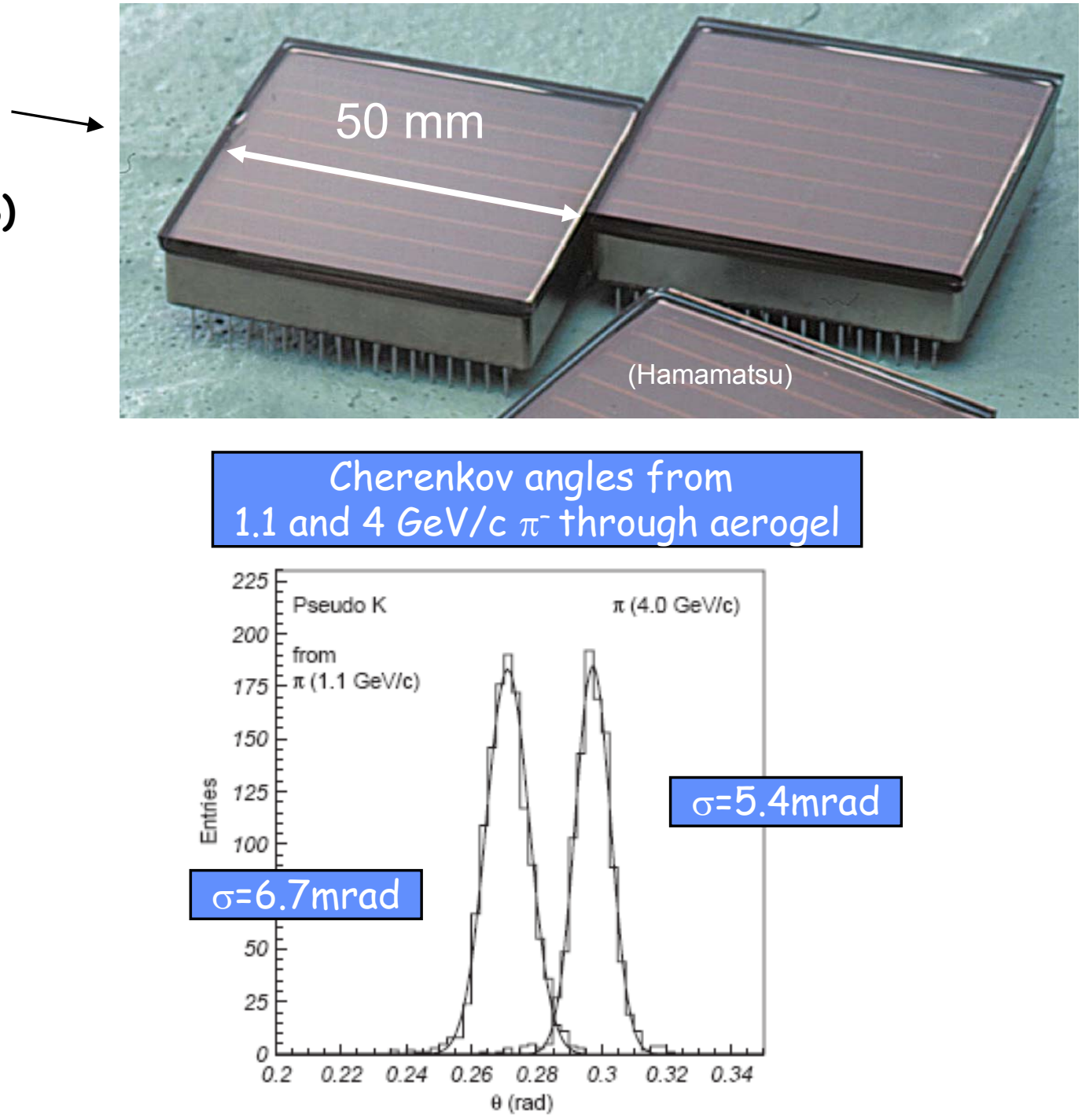

(P. Križan et al., NIM A 553 (2005) 58)

T. Gys - Vacuum photon detectors - RICH 2007 


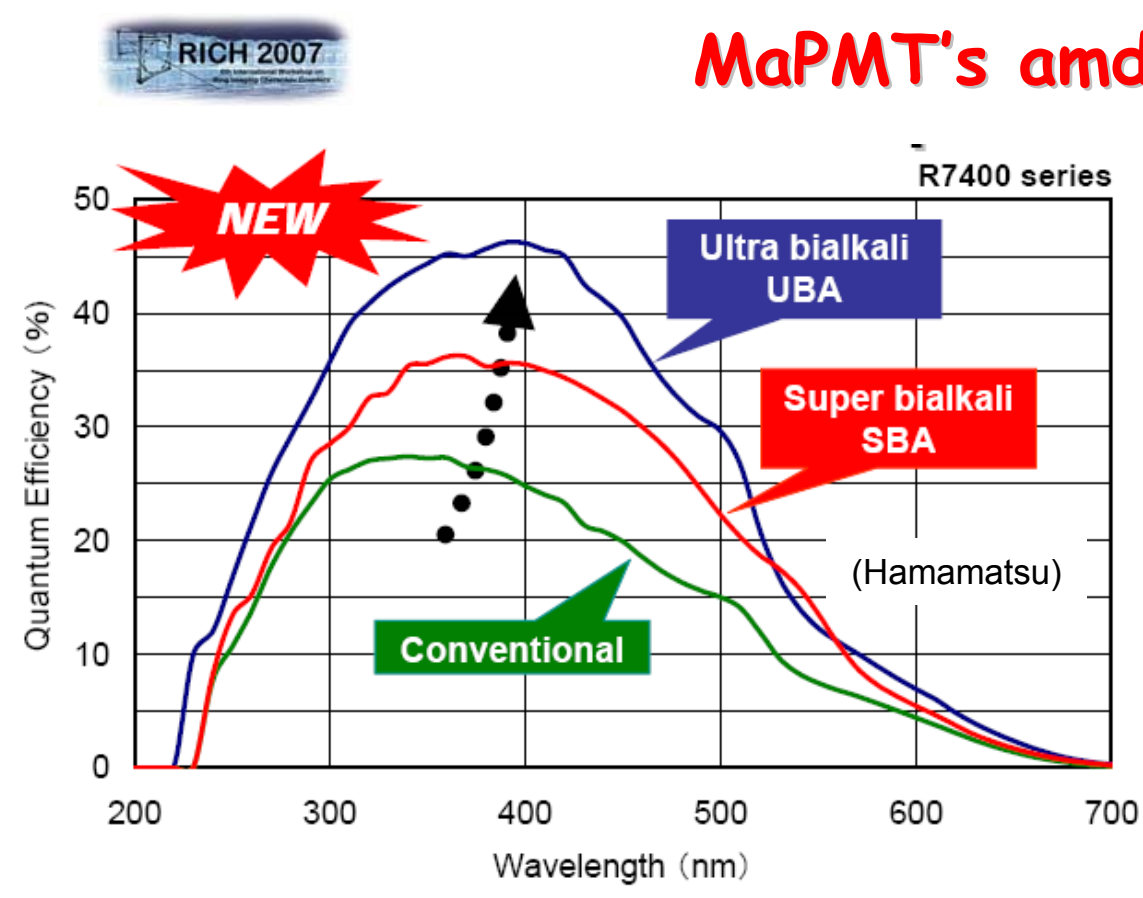

Improved gain uniformity of flat

jPET-D4 brain scanner GSO crystals
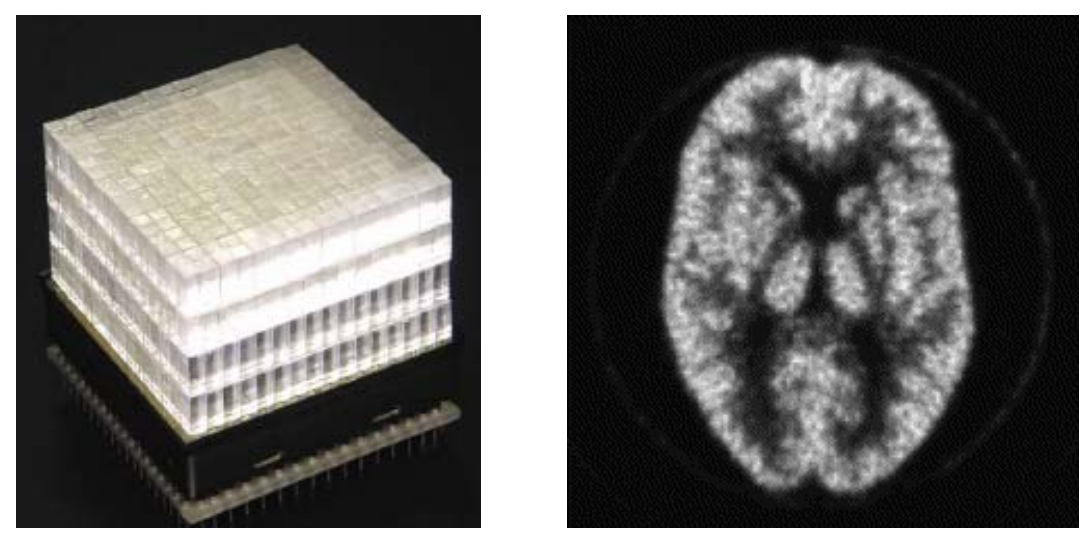

Improved bialkali photo-cathodes

- SBA:

- Metal package PMTs

- 1"-3" glass bulb PMTs

- UBA:

- Metal package PMTs panel metal package PMTs

- Typ.:

$$
\text { - } 1: 3 \Rightarrow 1: 2
$$

- Max.:

- $1: 5 \Rightarrow 1: 4$

T. Gys - Vacuum photon detectors - RICH 2007 
Barrel PID upgrade for super B factory - TOP Cherenkov counters (Nagoya, KEK)

See contributions of $K$. Inami,

P. Križan and A. Lehmann at this Conference

(M Akatsu et al., NIM A 440 (2000) 124)

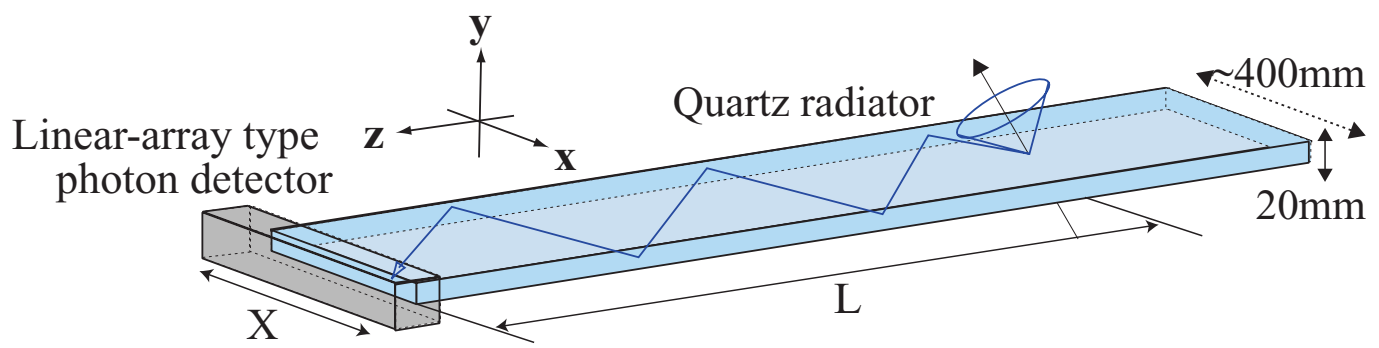

- Requirements

- Single photon sensitivity

- Good transit time spread (TTS<50ps)

- Immunity to high (1.5T) B-field

- Position-sensitive ( $5 \mathrm{~mm}$ )

- High detection efficiency

$\Rightarrow$ Best candidate is MCP-PMT

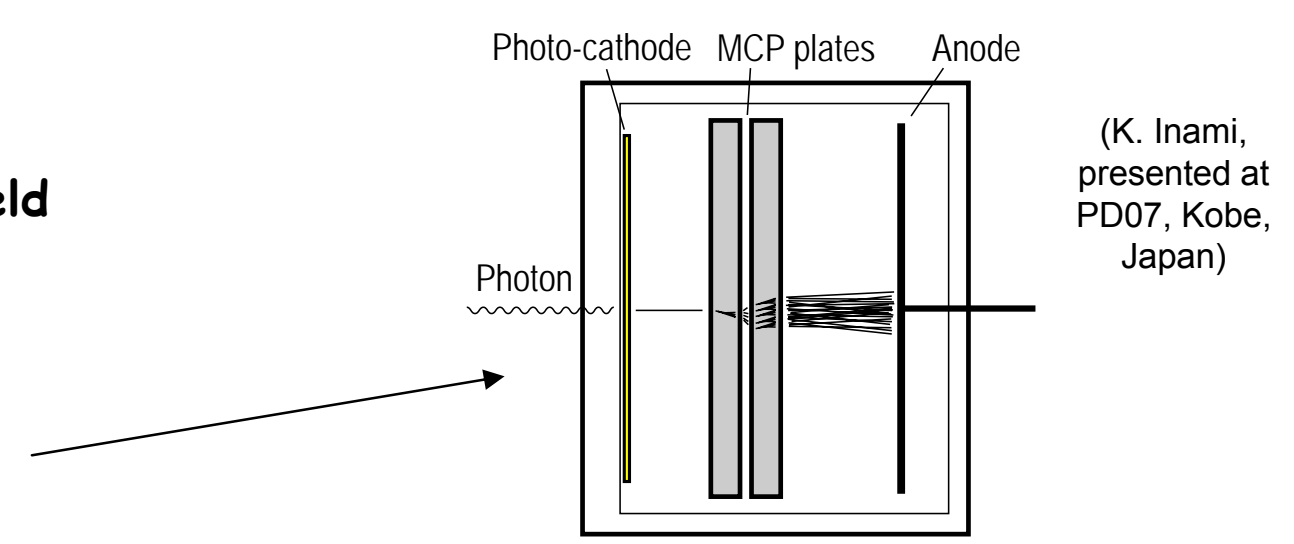


Super B factory upgrade (cont'd)

- MCP-PMT main features

- B-field immune due to small (6$25 \mu \mathrm{m}$ ) hole diameter - aperture typ. $60 \%$

- Excellent TTS (30ps for single photons at high gain)

- Photo-cathode (QE) ageing reduced with Al protective layer but CE drops from typ. 60 to $40 \%$

(N. Kishimoto et al., NIM A 564 (2006) 204)
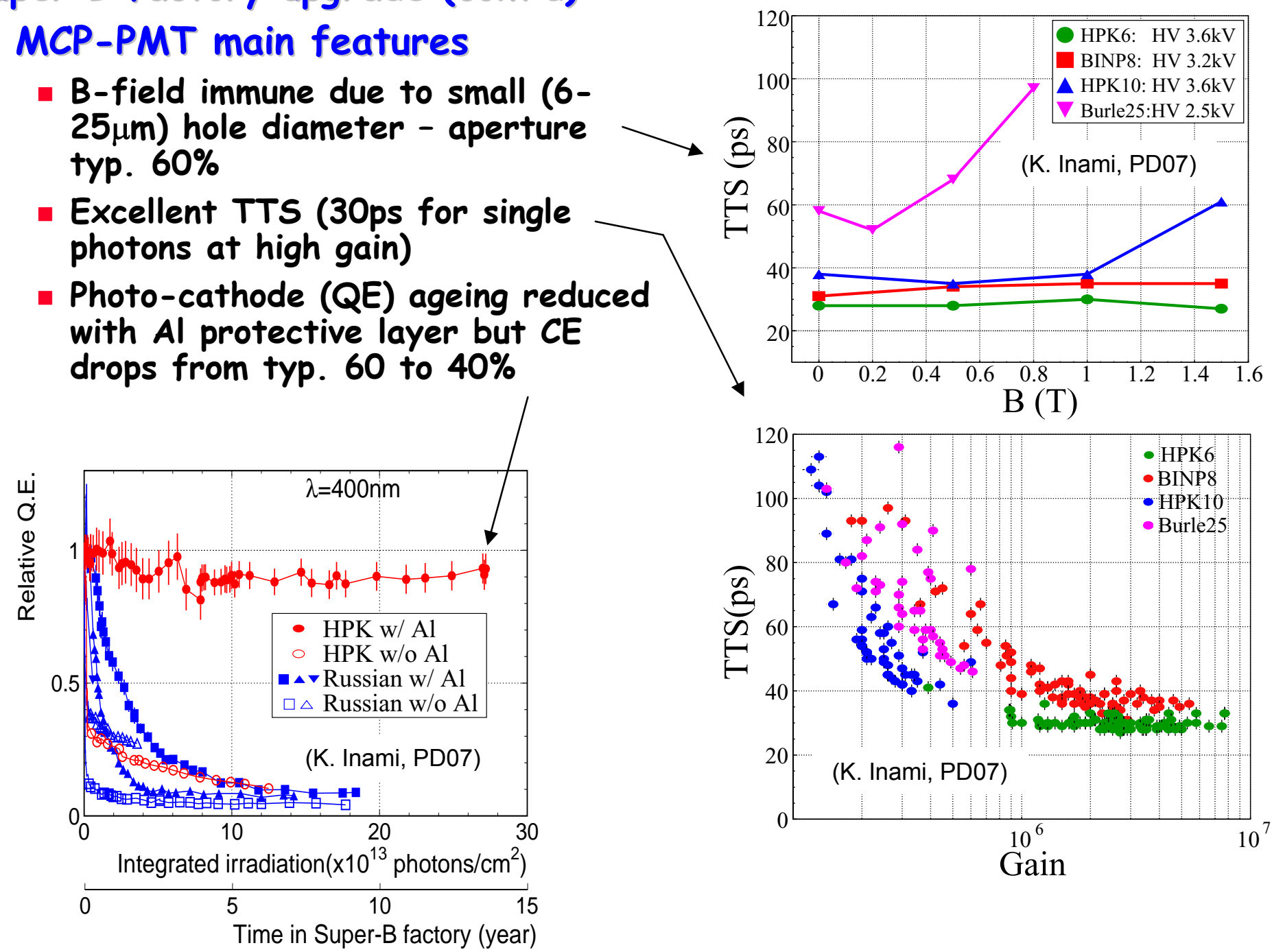

(M Akatsu et al. NIM A 528 (2004) 763)

T. Gys - Vacuum photon detectors - RICH 2007 


\section{MCP-PMT's: R\&D}

- Multi-anode MCP-PMT

- Large surface coverage (64\%)

- Linear position information $(4 \times 5.3 \mathrm{~mm} \times 22 \mathrm{~mm})$

- Fast rise time (400ps)

- Excellent TTS (30ps for single photons)
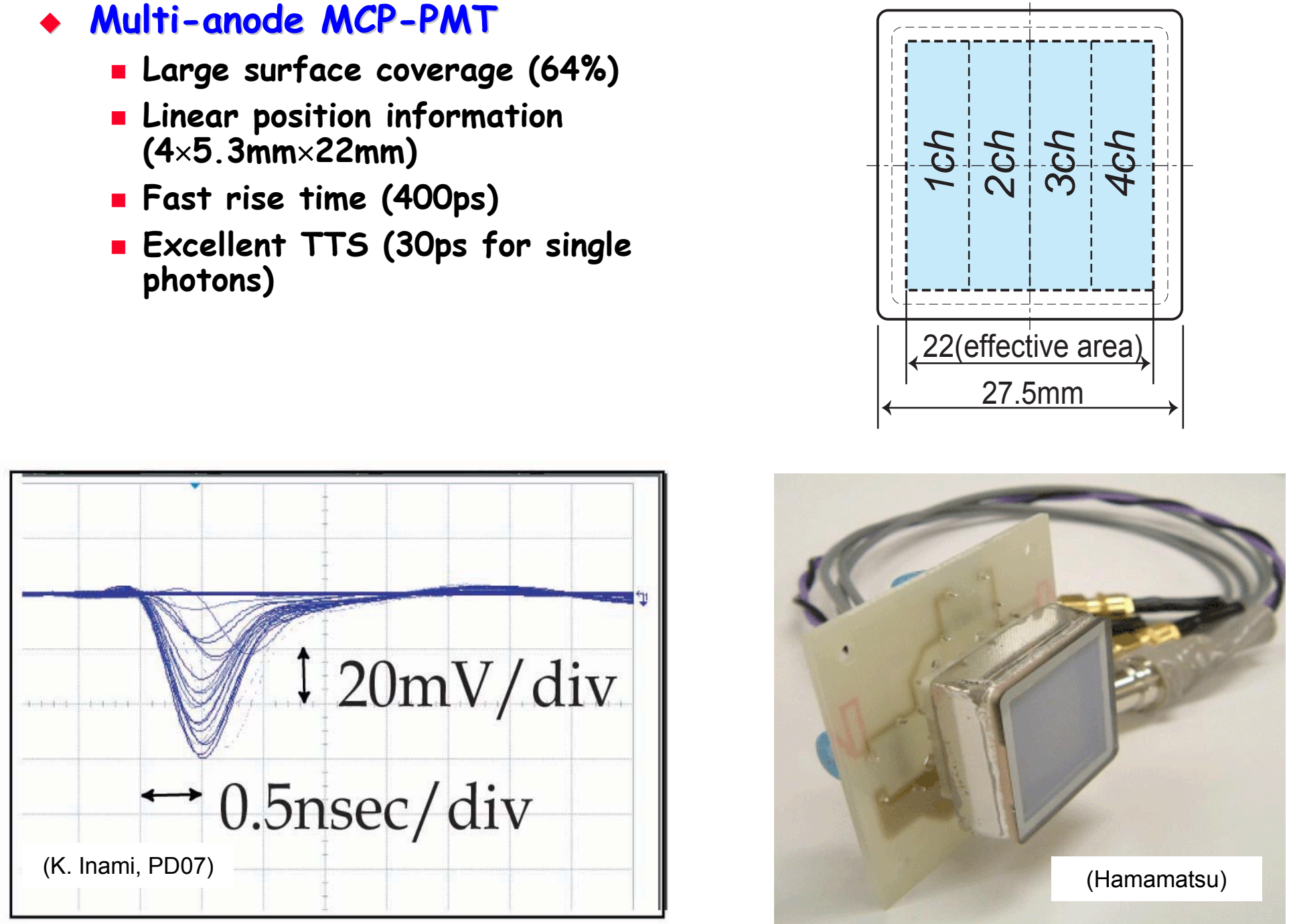

T. Gys - Vacuum photon detectors - RICH 2007 
Main features

- Construction

- Hybrid technology: vacuum photon detector tube encapsulating a solid-state detector (+ possibly its readout electronics)

- Segmentation ranges from $\sim 50 \mu \mathrm{m}$ to $\sim 20 \mathrm{~mm}$

- Various possible e-optics designs based on image intensifier technology

- Gain $\cong 1$ to $5 \times 10^{3}$

- Small intrinsic fluctuations $\cong \sqrt{ } F \times G$ + back-scattering effects

$\Rightarrow$ overall noise dominated by electronics noise

- Gain uniformity typ. 1

- Cross-talk: see CMS HCAL
(C.A. Johansen et al., NIM A 326 (1993) 295-298)

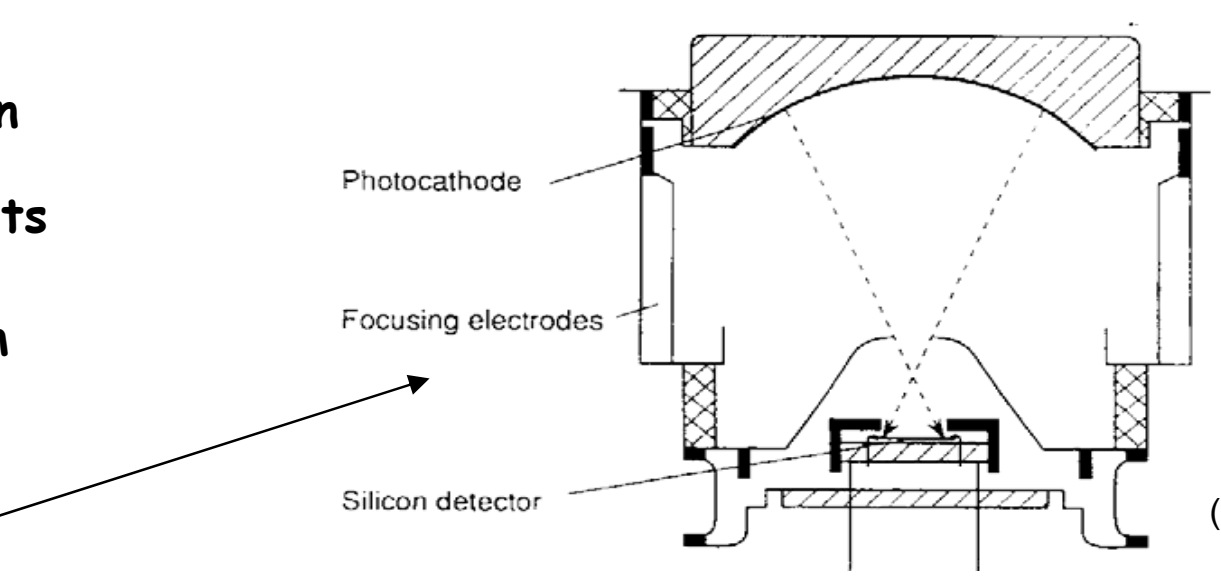

(PhotonisDEP)

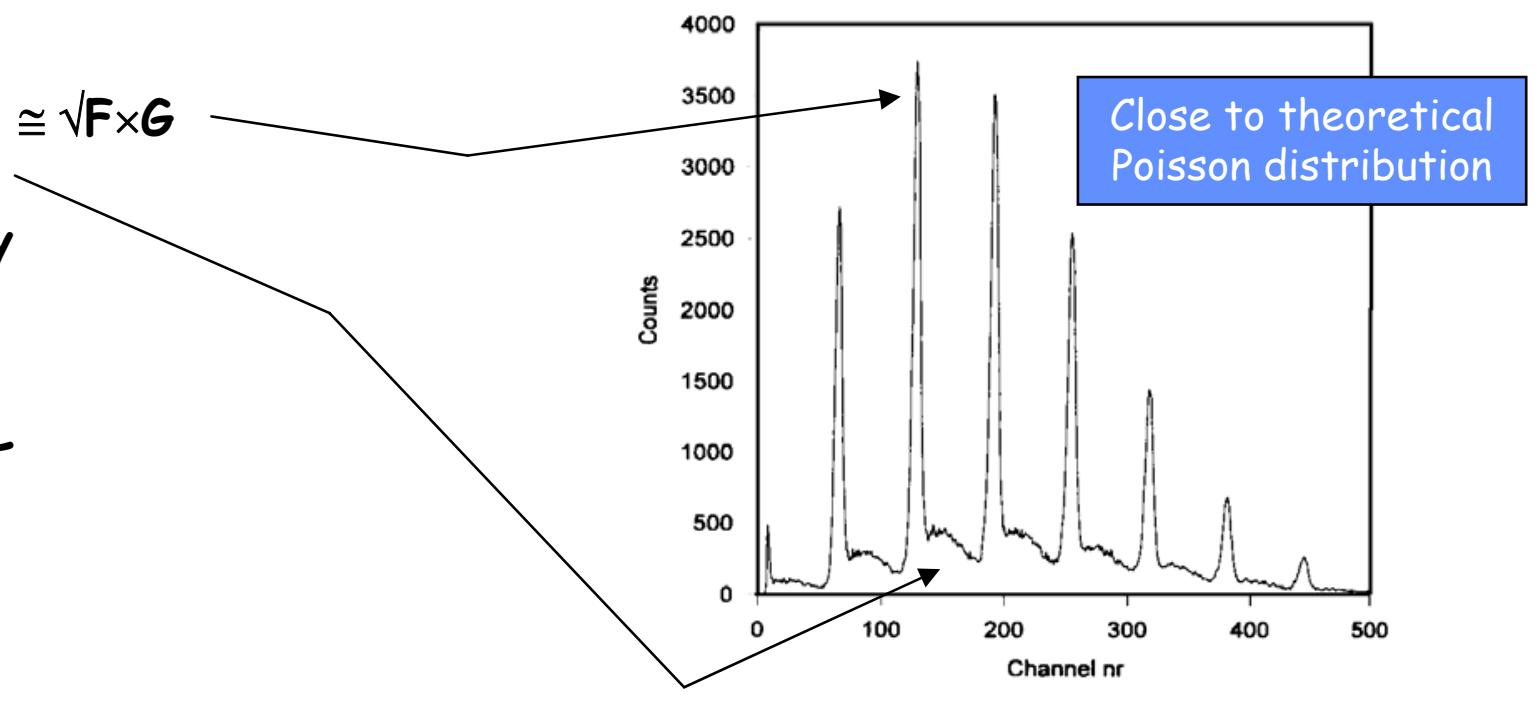

(C.P. Datema et al., NIM A 387 (1997) 100-103)

T. Gys - Vacuum photon detectors - RICH 2007 


\section{HPD's (2)}

HPDs for LHCb RICH detectors

- Requirements

See contributions of $S$. Eisenhardt and S. Brisbane at this Conference

- Large area (3.3m²) with high overall active area fraction ( $65 \%$ )

- Fast compared to the 25 ns bunch crossing time

- Have to operate in a small (1-3mT) magnetic field

- Granularity $2.5 \times 2.5 \mathrm{~mm}^{2}$

$\Rightarrow 484$ HPDs with $5 x$ demagnification and custom anode
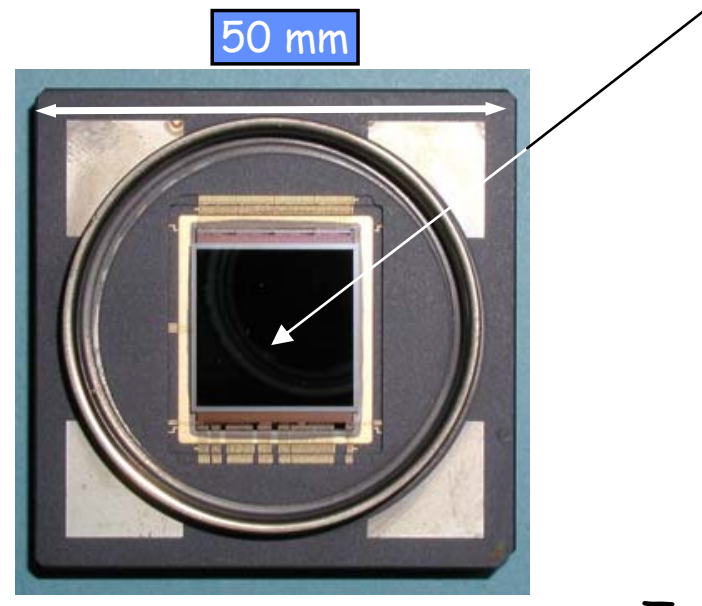

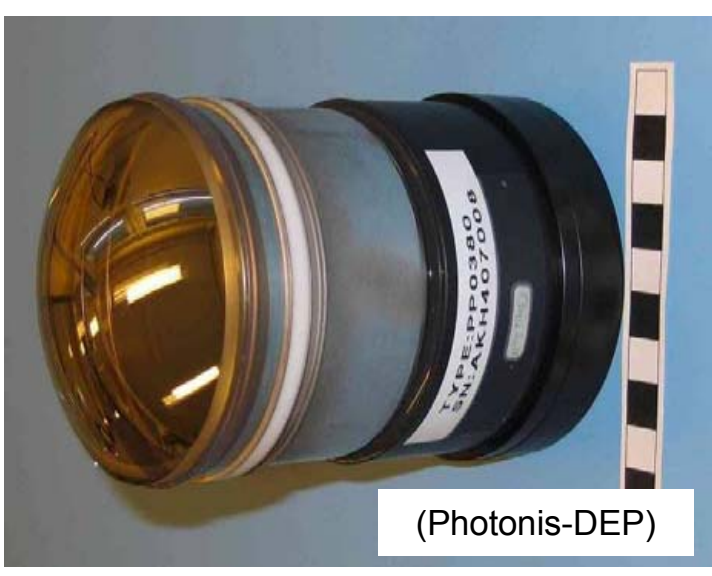

T. Gys - Vacuum photon detectors - RICH 2007
Si pixel array (1024 elements)

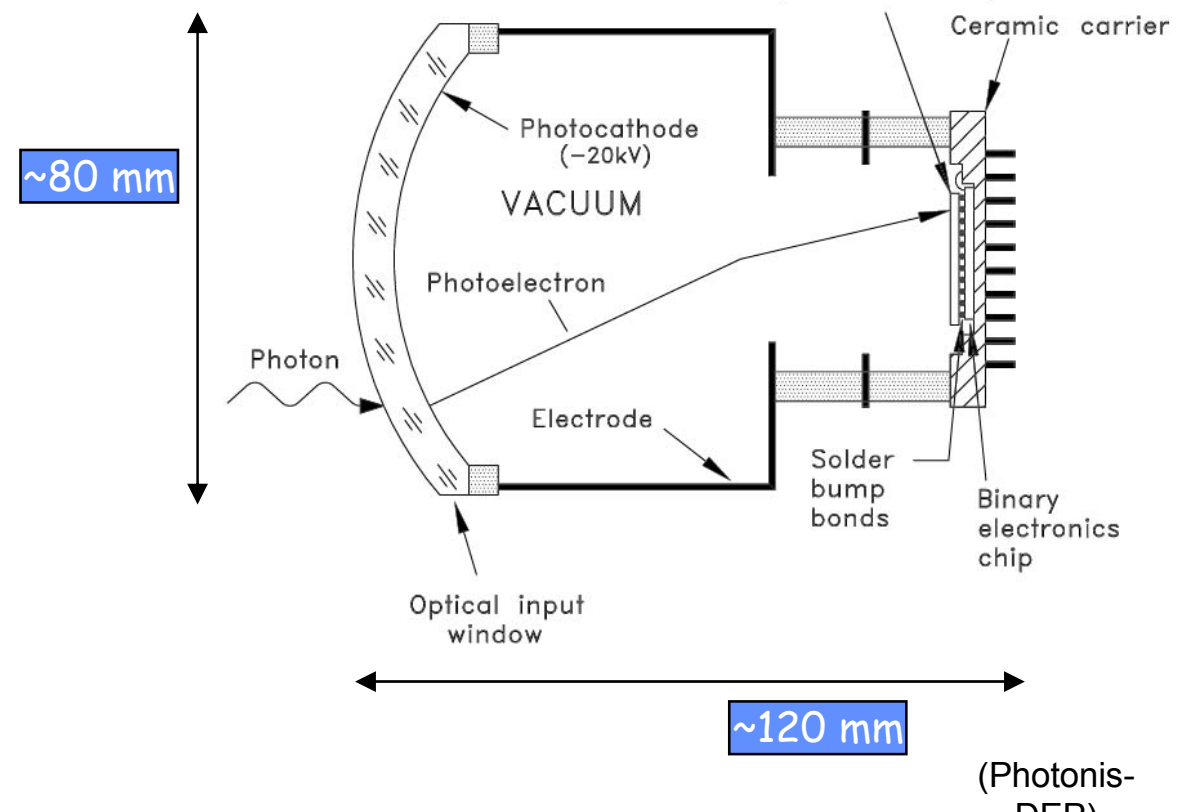

DEP)

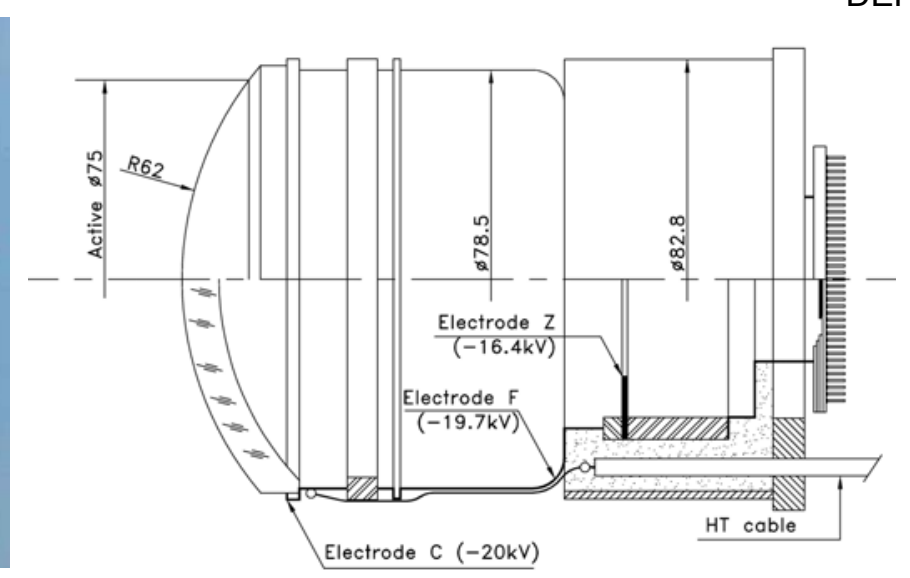




\section{HPD's (3)}

HPDs for LHCb RICHes (cont'd)

- HPD photo-cathode

- Must cover 200-600nm wavelength range

- Multi-alkali S2O (KCsSbNa ${ }_{2}$ )

- Improved over production

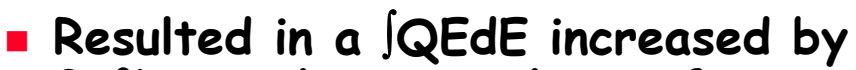
$27 \%$ wrt the original specifications
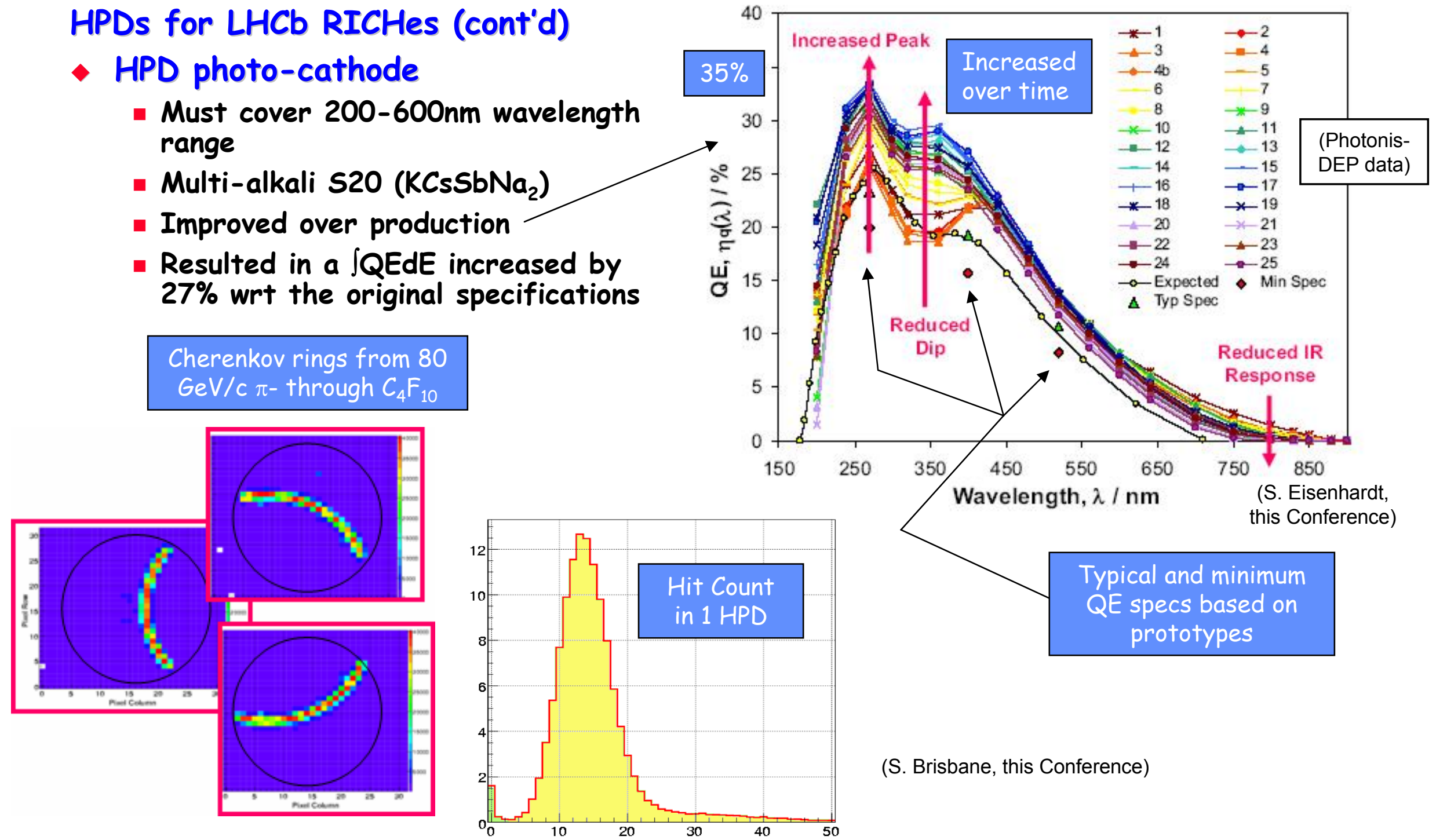

T. Gys - Vacuum photon detectors - RICH 2007 
HPDs for LHCb RICHes (cont'd)

- Thresholds and noise

- The anode is a Si pixel detector with 8192 channels organized in 1024 super-pixels of $500 \times 500$ $\mu \mathrm{m}^{2}$ size, bump-bonded to a custom binary readout chip (Ihcbpix1)

$\Rightarrow$ excellent signal-to-noise ratio achieved by small pixels and optimal sensor-FE coupling

- Very low average threshold and noise

- Typical signal is 5000 e(Si detector dead layer typ. $150 \mathrm{~nm}$ ) with intrinsically low fluctuations (typ. $25 e-r m s$ )

$\Rightarrow \sim 85 \%$ photo-electron detection efficiency for 25 ns strobe

- Residual inefficiency is dictated by photo-electron back-scattering (18\% probability) and chargesharing effects

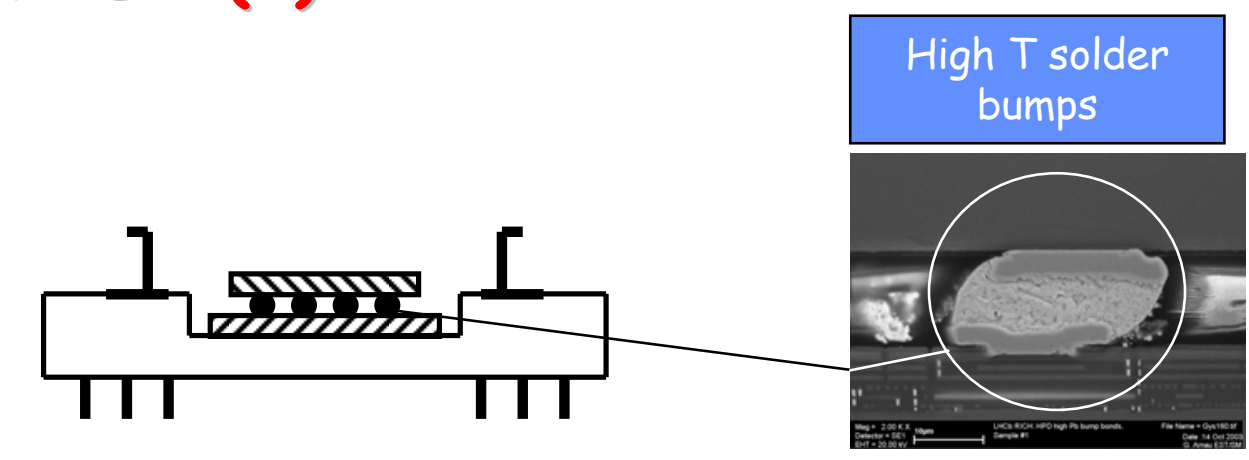

(M. Campbell et al., IEEE TNS 53, 4 (2006), 2296)

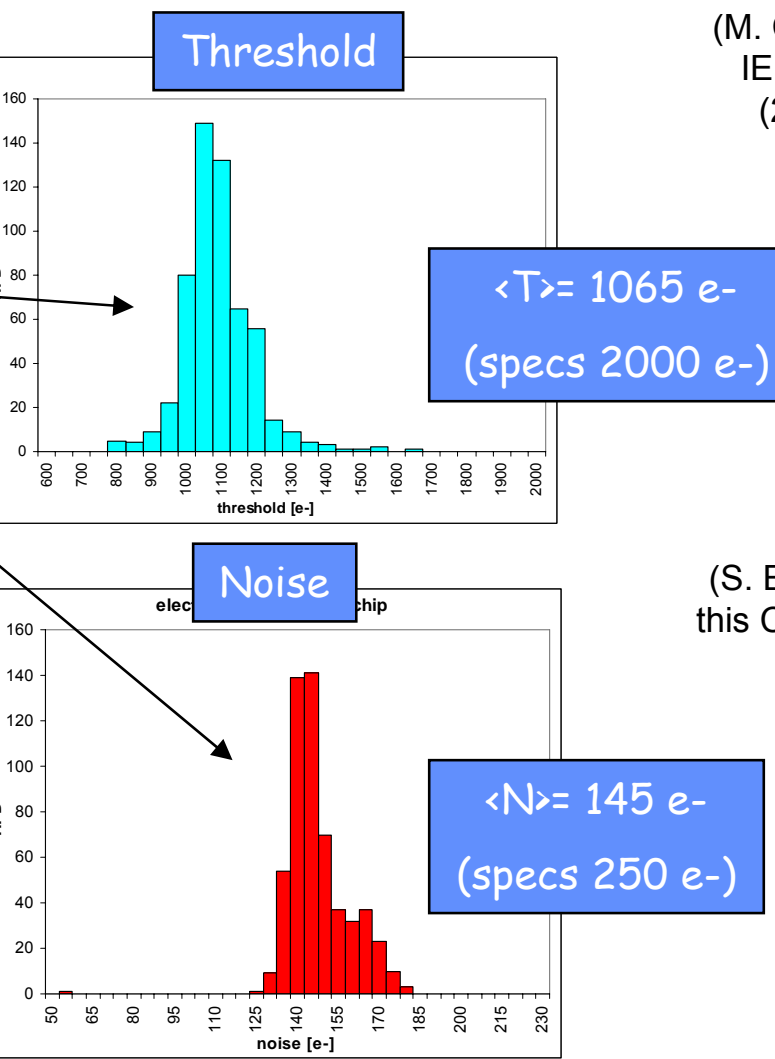

T. Gys - Vacuum photon detectors - RICH 2007 
HPDs for LHCb RICHes (cont'd)

- Local magnetic shielding

- To avoid image loss and minimize distortions, local shielding of HPD's required to reduce $B$ field below $106(1 \mathrm{mT})$ inside HPD volume

- With test pattern, reconstruct pixel hit - photon hit position correspondence for each HPD

- Distortion correction must not degrade pixel size error

Mu-metal shield grounded and insulated with 3 layers of $125 \mu \mathrm{m}$-thick Kapton foil
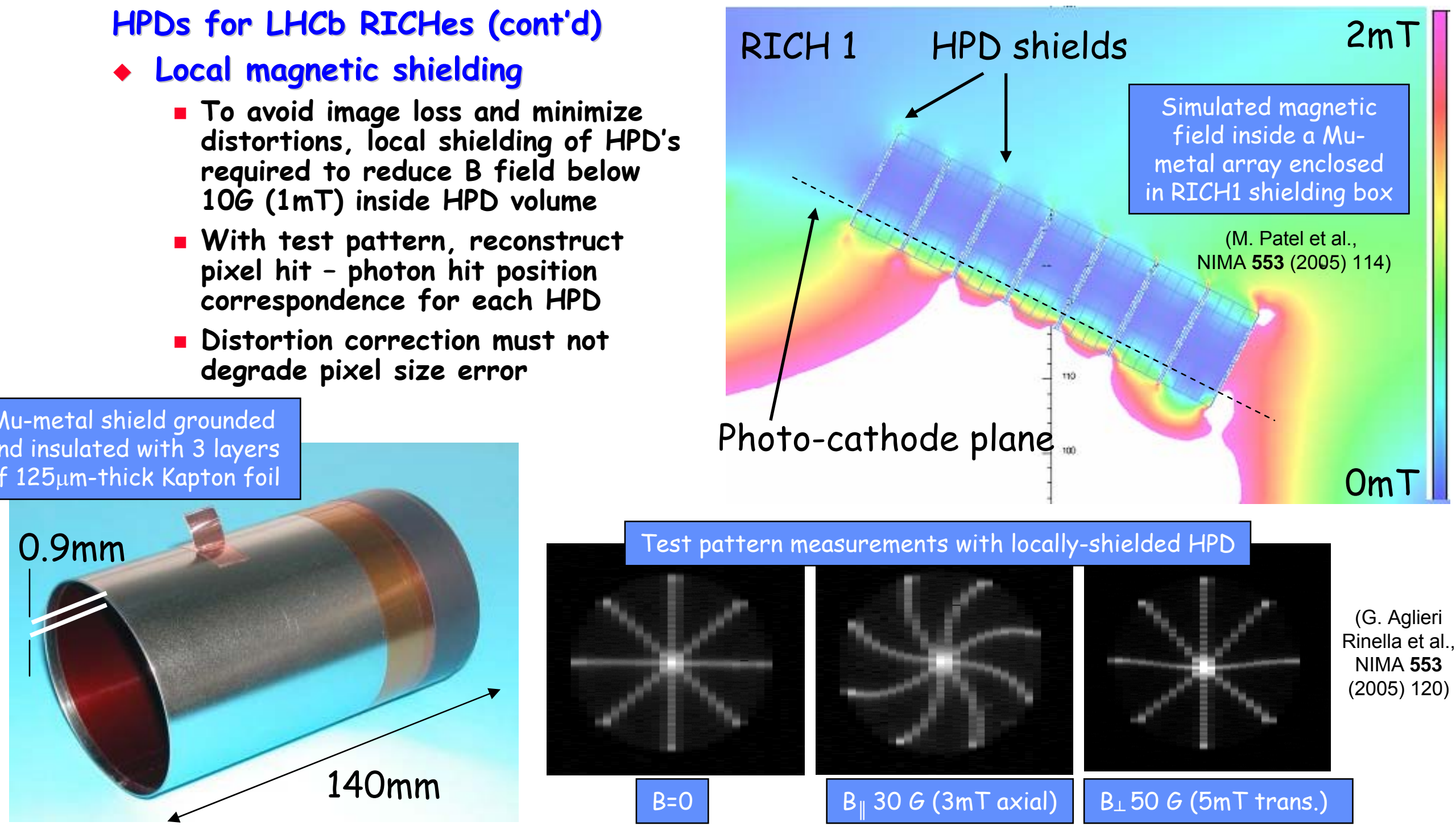

T. Gys - Vacuum photon detectors - RICH 2007 


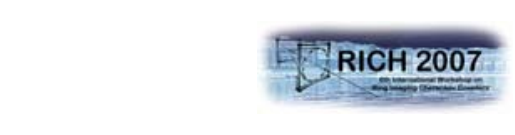

\section{Multi-pixel proximity-focussed HPD}

\section{CMS HCAL}

- Requirements

- $B=4 T \Rightarrow$ proximity-focussing with $3.35 \mathrm{~mm}$ gap and $\mathrm{HV}=10 \mathrm{kV}$;

- Minimize cross-talks:

- pe back-scattering: align with B:

- capacitive: Al layer coating:

- internal light reflections: a-Si:H AR coating optimized @ $\lambda=520 \mathrm{~nm}$ (WLS fibres):

Layer to Tower Decoding Fiber

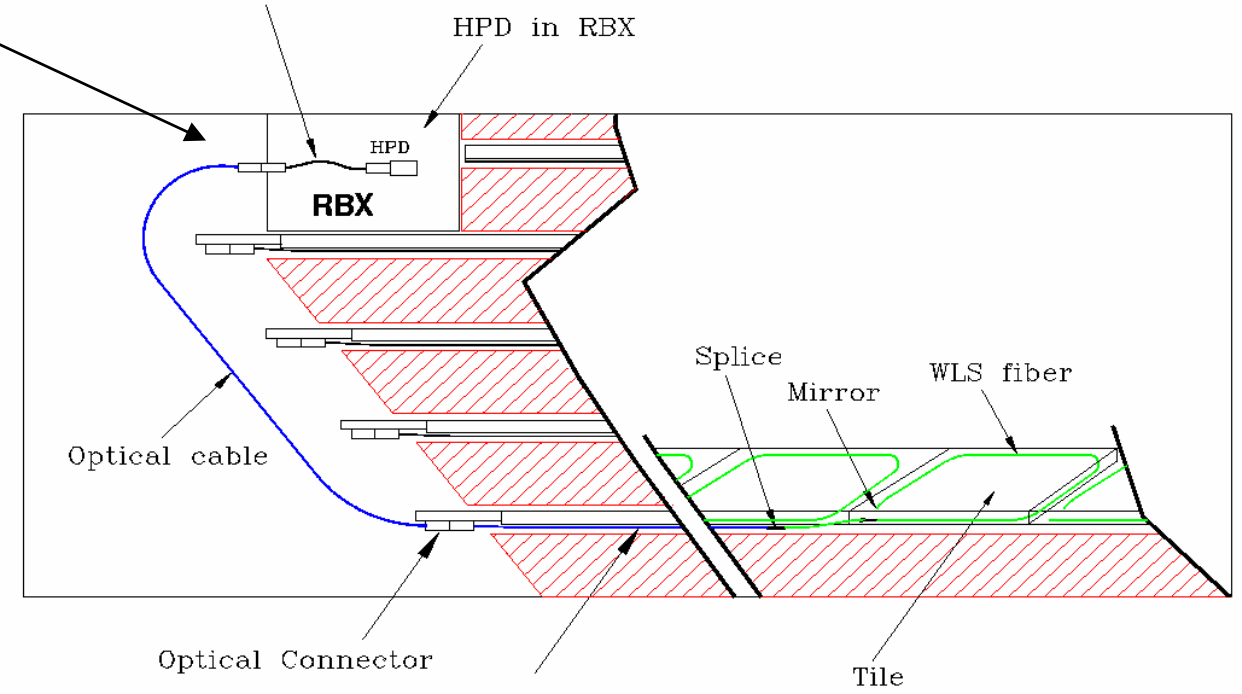

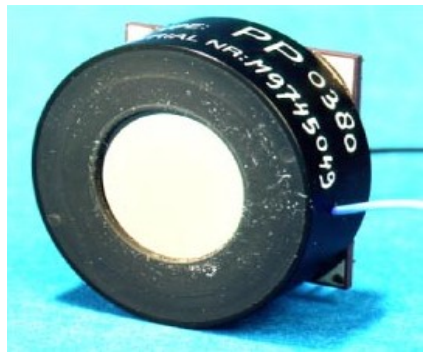

(Photonis-DEP)

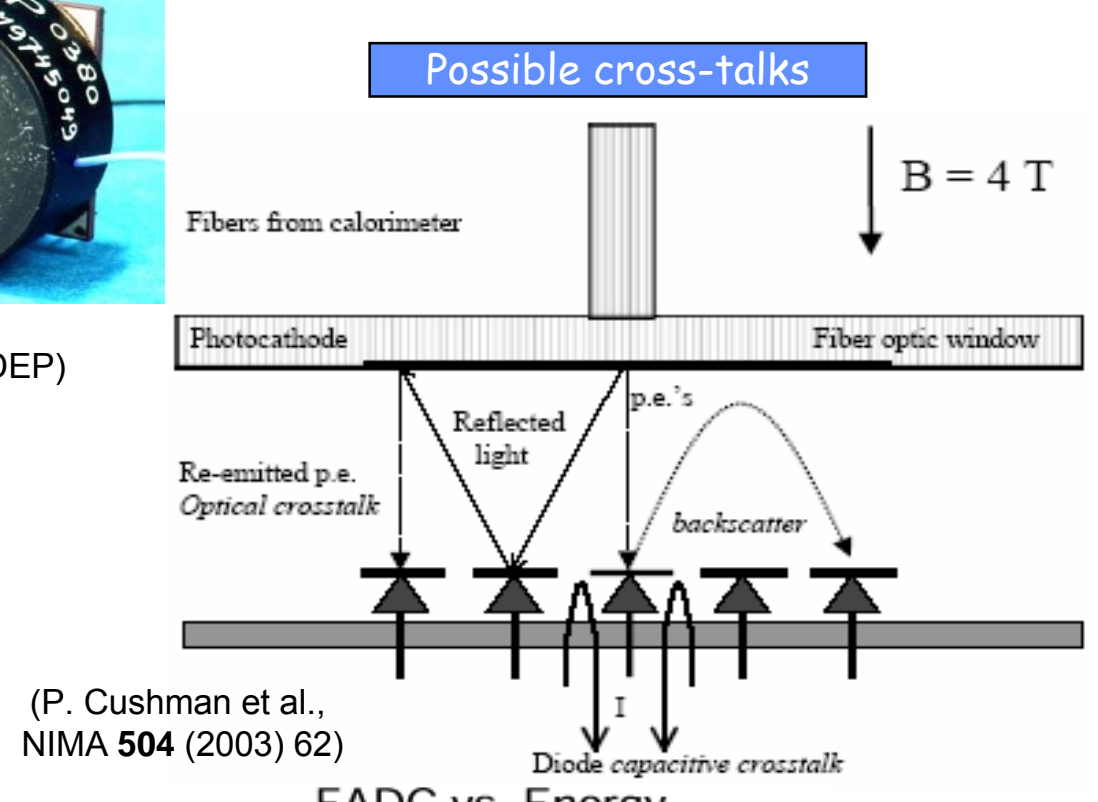

FADC vs. Energy

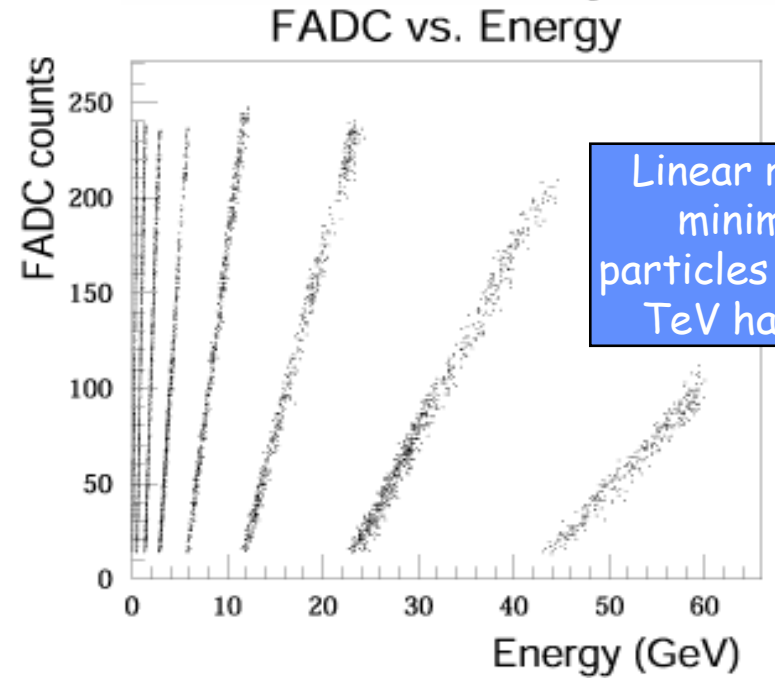

T. Gys - Vacuum photon detectors - RICH 2007 


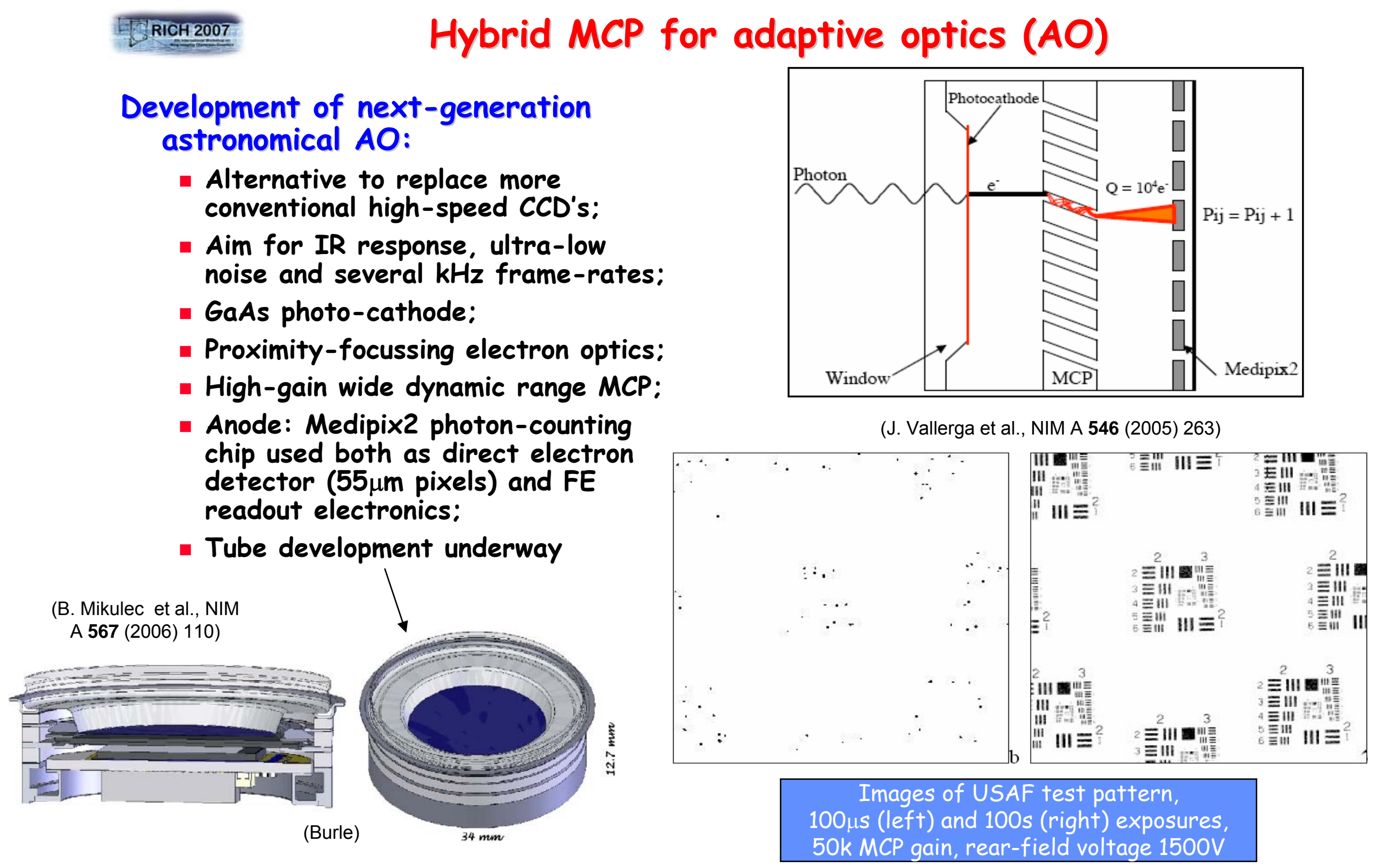

T. Gys - Vacuum photon detectors - RICH 2007 
Aerogel RICH belle Upgrade

See contribution of S. Nishida at this Conference

- Construction

- 4 chips of $6 \times 6$ APD each $5 \times 5 \mathrm{~mm}^{2}$

- Immune to B-fields up to 1.5T

- Active area ratio $64 \%$

- HV typ. 10kV, V typ. 300V

- Gain typ. $1000 \times 10$

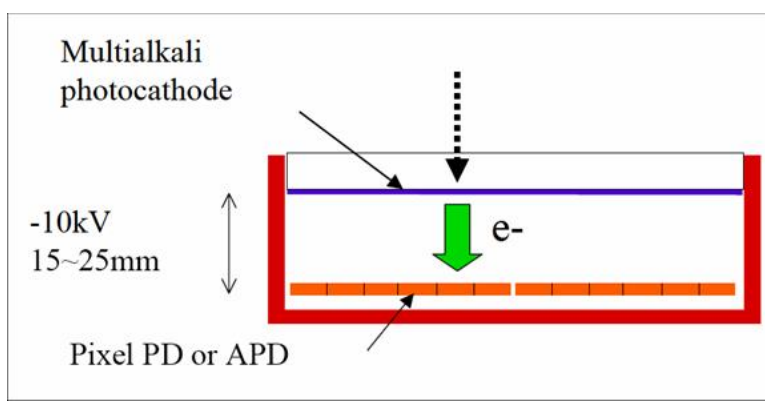

(I. Adachi, presented at PD07, Kobe, Japan)

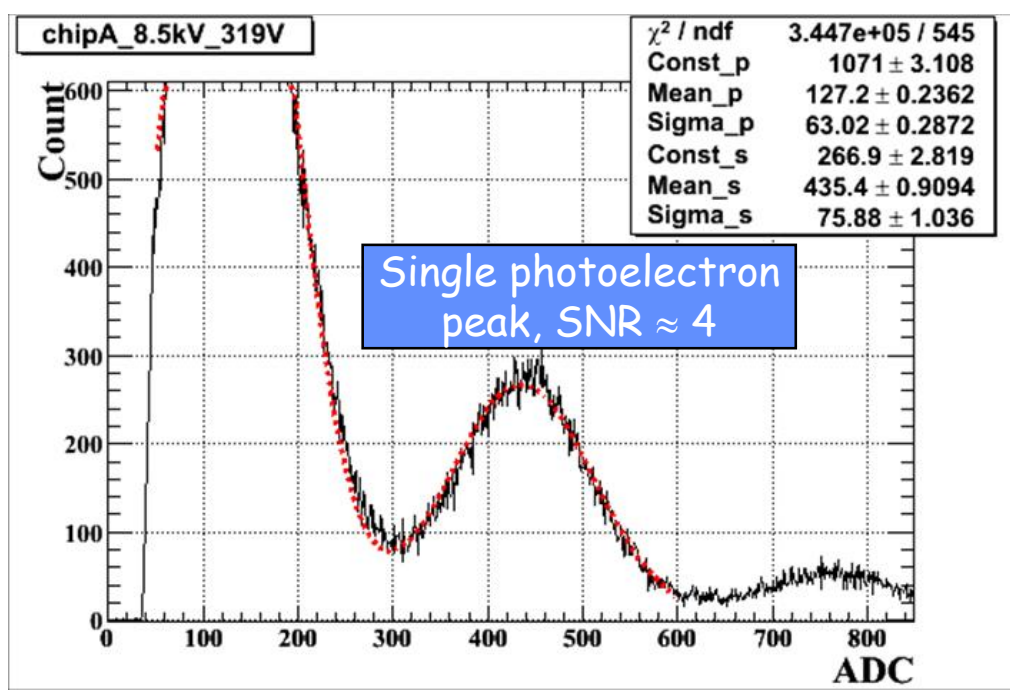

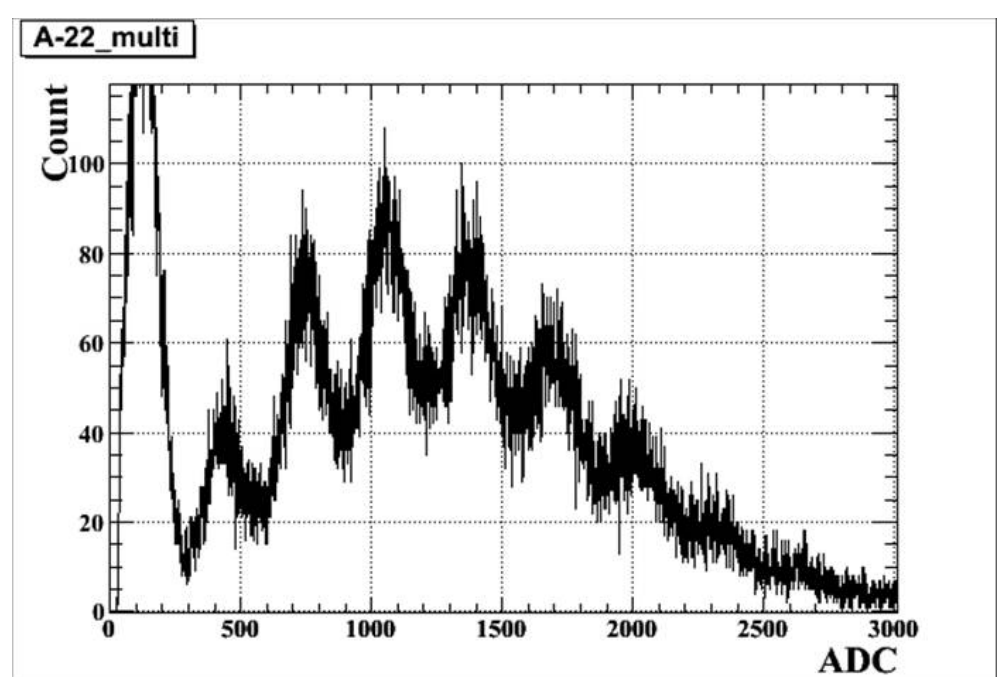

T. Gys - Vacuum photon detectors - RICH 2007 


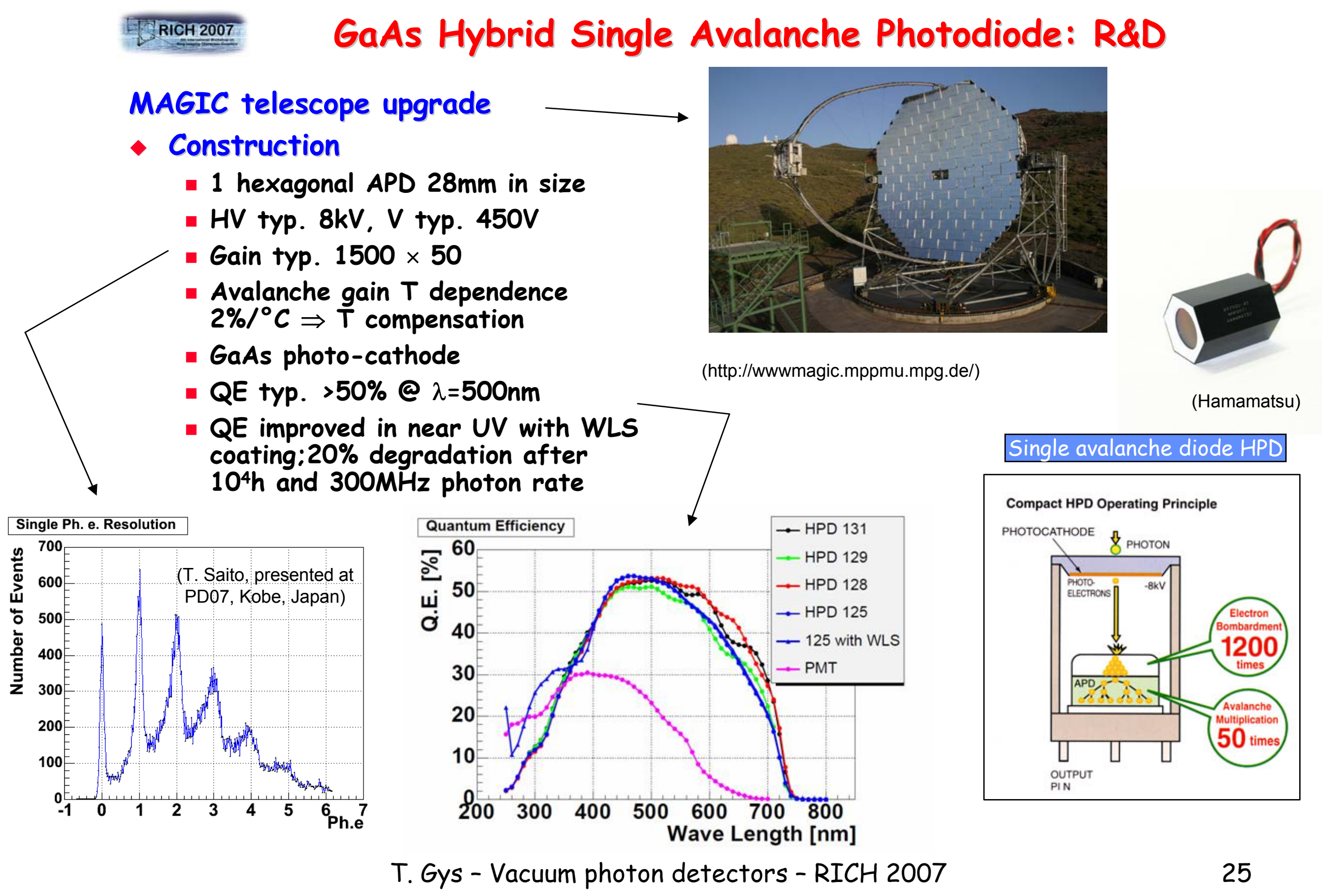




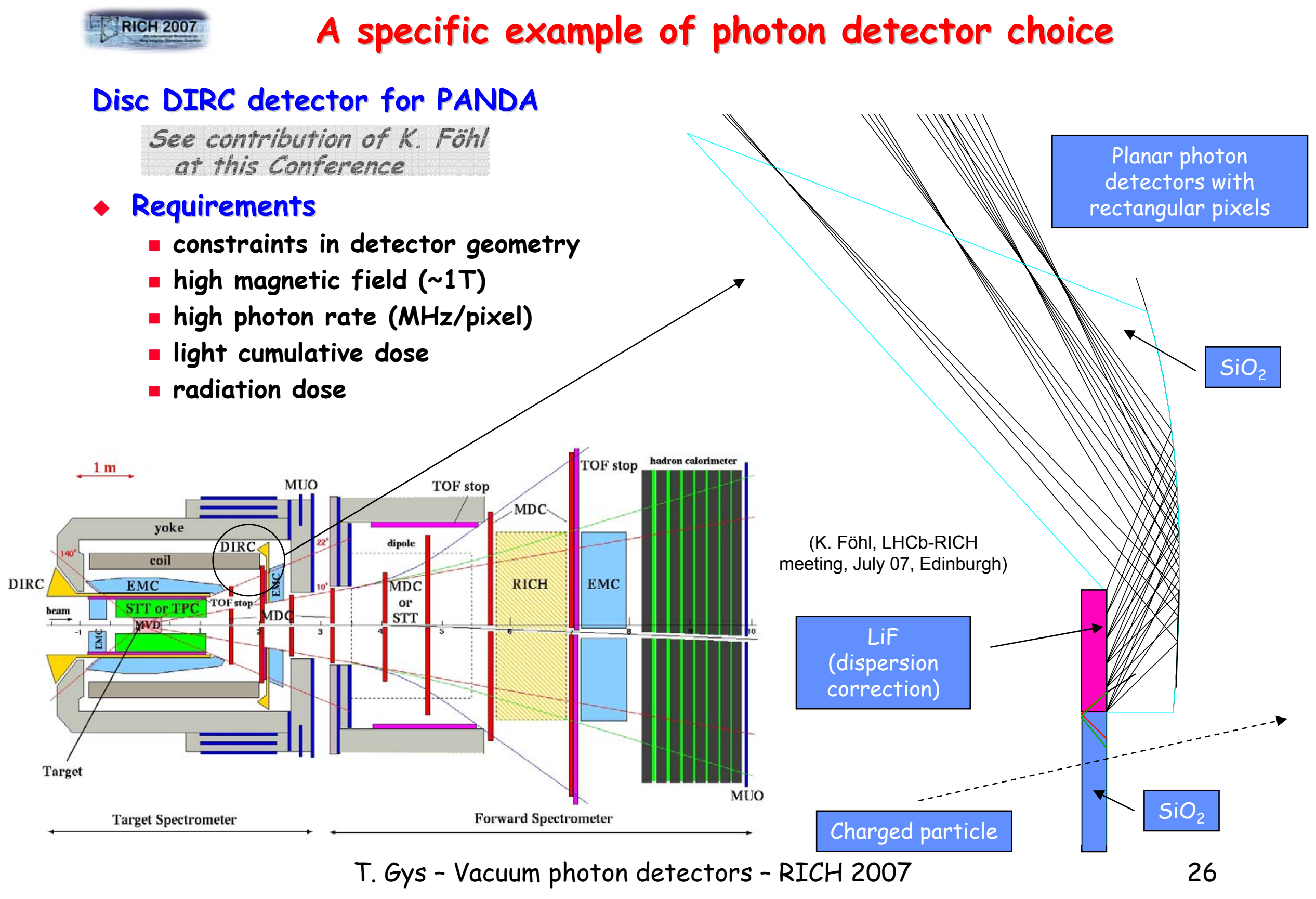


Disc DIRC detector for PANDA (cont'd)

- Photon detector options:

- position-sensitive PMT's

- B-field immunity

- gain uniformity

- APDs or similar (MPPC, SiPM, etc.)

- dark noise vs high signal rate

- radiation hardness

- channel plate phototubes

- overall single pe- efficiency

- optical fibres and external phototubes

- fibre connections and related losses

- HPDs with electro-magnetic imaging

- alignment with B field

- radiation hardness

- Readout electronics?

(K. Foehl, LHCb-RICH meeting, July 07, Edinburgh)

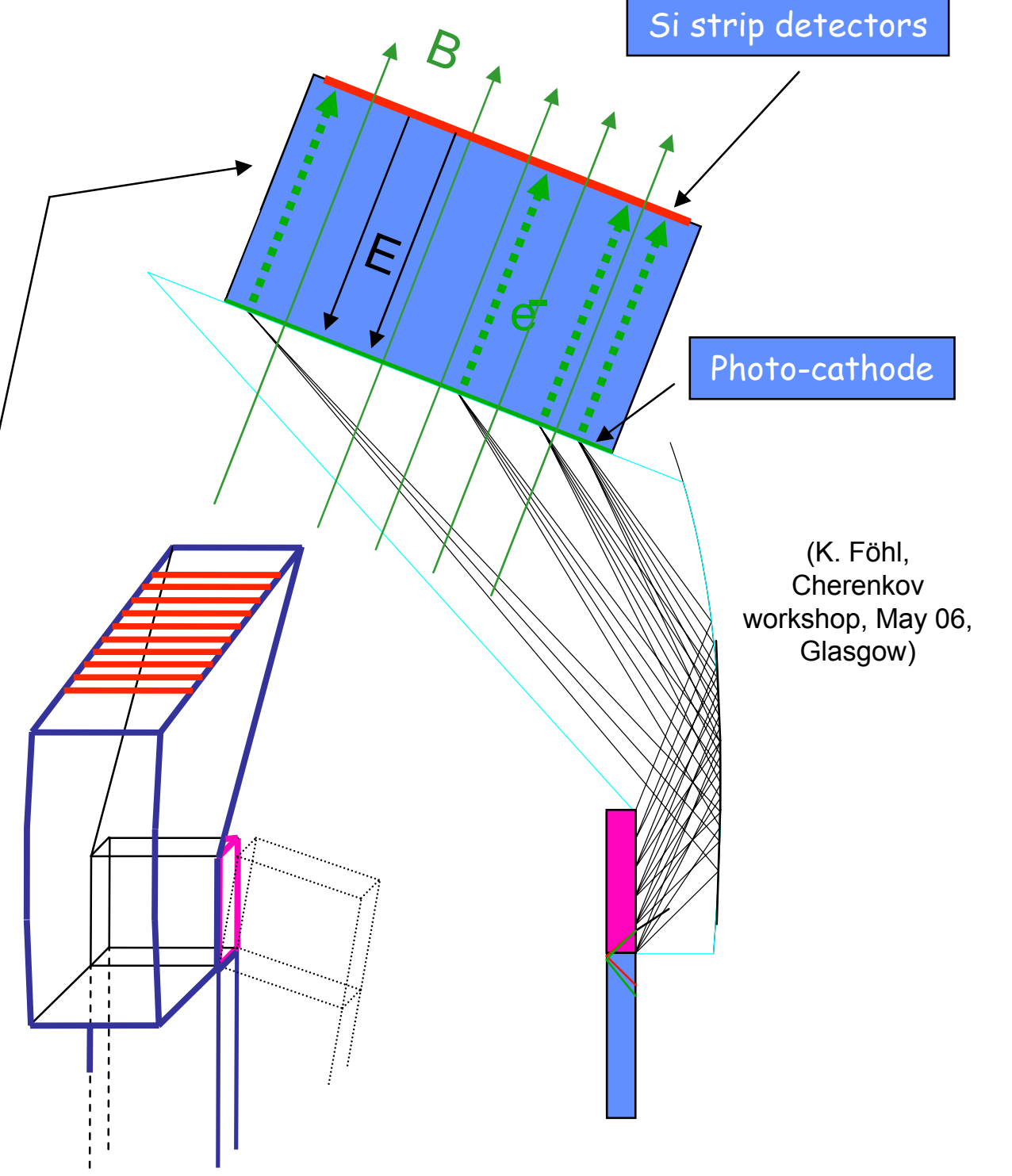

T. Gys - Vacuum photon detectors - RICH 2007 
- A lot of innovative techniques are being used or developed!

- There is room for improvement on many aspects, including supposedly routine aspects like quantum efficiency of photo-cathodes.

- Design aspects

- Dictated by very specific application requirements

- Trade-off between:

- granularity

- speed

- active surface

-...

- cost!

$\Rightarrow$ no fully optimal solution

- Design guidelines

- Survey of existing technologies

- Collaboration with industry $\Rightarrow$ as much as possible, try to combine/match requirements with industrial standards

- Development of new photon detectors and their associated readout (front-end) electronics should be carried out in parallel but not independently

T. Gys - Vacuum photon detectors - RICH 2007 